\title{
Wideband sound absorption and transmission through micro-capillary plates: Modelling and experimental validation
}

\author{
Cédric Maury ${ }^{a}$, Teresa Bravo ${ }^{\text {b, * }}$ \\ a Aix Marseille Univ, CNRS, Centrale Marseille, Laboratoire de Mécanique et d'Acoustique (LMA), 38 rue Frédéric Joliot-Curie, 13013 , \\ Marseille, France \\ ${ }^{\mathrm{b}}$ Instituto de Tecnologías Físicas y de la Información (ITEFI), Consejo Superior de Investigaciones Científicas (CSIC), Serrano 144, 28006, \\ Madrid, Spain
}

\section{A R T I C L E I N F O}

\section{Article history:}

Received 24 October 2019

Received in revised form 10 March 2020

Accepted 28 March 2020

Available online 8 April 2020

Handling Editor: D. Juve

\section{Keywords:}

Micro-capillary plates

Sound absorption

Anechoic termination

\begin{abstract}
A B S T R A C T
The current work presents theoretical and experimental studies that investigate sound propagation through micro-capillary plates (MCP) under a general plane wave excitation and in the no-flow case. MCPs are characterized by micrometric channels radius with Knudsen number greater than 0.001 so that a slip-flow model has been derived for their viscous transfer impedance. It is found that the slip-flow model should be used instead of the continuum model to predict the transfer impedance of MCPs with channels radii lower than $2 \mu \mathrm{m}$ as well as their absorption coefficient under near-grazing incident excitations. Otherwise, both approaches provide similar results, as confirmed by comparison with finite element simulations. Due to their high porosity, MCPs provide minute reactance and constant resistance that can be tailored to achieve target absorption over a broad frequency range. Plane wave impedance tube experiments have shown that a near-optimal MCP termination can provide a low frequency flat absorption spectrum that stays above 0.7 up to a Helmholtz number of 1.84. Measurements on rigidly-backed MCPs have led to ultra-wideband absorption with a half-bandwidth spanning up to 12 octaves around the absorber Helmholtz resonance. Expressions have been derived to find the optimal channels radius that maximize the MCPs dissipation under general incidence angle and assuming anechoic or rigid backing. The sensitivity of the MCPs optimal transfer resistance to their load impedance has been examined. It provides a design chart to find the MCP optimal parameters that achieve specific broadband absorption value under general incidence and practical load conditions.
\end{abstract}

(c) 2020 Elsevier Ltd. All rights reserved.

\section{Introduction}

Micro-perforated panel (MPP) absorbers are noise control solutions that have been extensively studied due to their promising applications in a wide range of areas, such as room acoustics [1] and noise barriers [2], but also as windows [3] and duct [4] silencing systems in order to attenuate sound transmission whilst preserving flow circulation. MPPs do not emit

\footnotetext{
* Corresponding author.

E-mail addresses: cedric.maury@centrale-marseille.fr (C. Maury), teresa.bravo@csic.es (T. Bravo).
} 
airborne fibers, may withstand severe environmental conditions and can be made of lightweight and transparent materials. When insonified, the use of sub-millimeter perforations significantly increases the air particle velocity through the holes and so the visco-thermal dissipation of acoustical energy into heat. When the viscous boundary layer thickness is much lower than the holes radius, another source of absorption is the generation of acoustically-induced vorticity at the holes inlet/outlet [5].

A first category of absorbents concerns MPP resonance absorbers backed by a rigid air cavity. Maximum absorption occurs at the Helmholtz-type resonance whose half-bandwidth typically extends between one and two octaves around the peak frequency [6,7]. In order to broaden the bandwidth without altering the maximum absorption value, a multi-layered distribution of MPPs and air cavities have been considered with a number of distinct Helmholtz resonances that scales on the number of MPPs [8,9]. Decreasing the perforation ratio of the MPPs from the source side to the rigid backing enhances the absorption as it creates a positive gradient of flow resistance $[2,10]$.

In order to better comply with constraints on the overall thickness of the absorbent, parallel distributions of rigidly-backed MPP resonators with different cavity depths or perforation ratios have been studied [11,12]. They showed wideband absorption performance caused by cross-coupling mechanisms between the resonators. Compound serial-parallel arrangements of rigid [13] or elastic [14] MPP absorbers were found to further extend the bandwidth to higher frequencies. Other strategies such as extended rigid [15] or flexible [16] tubes in the cavity are able to downshift the Helmholtz-type resonances to lower frequencies due to an increase of the effective acoustic path length. Note that the above absorbents build upon Helmholtz-type resonances to achieve, under normal incidence and in the no-flow case, impedance matching with the air over a bandwidth as wide as possible. A similar challenge has also been pursued considering arrays of quarter-wavelength resonators with coiled-space cavities [17]. In all cases, a trade-off is achieved between the relative absorption bandwidth and the absorbent overall thickness that cannot fall below a certain threshold for a given absorption bandwidth due to causality constraint [18].

A second category concerns unbacked MPPs. They are able to absorb sound energy at low frequencies through their sole acoustical flow resistance. A single MPP in an enclosure was found to provide substantial attenuation of the cavity modes [19] and could be used to reduce boiler tones in heat-exchange cavities. Double-leaf MPPs [20] were shown to work both as resistive and Helmholtz-type absorbers at low and mid frequencies, respectively, and could be used as space absorbers in room interiors. Other configurations have shown the efficiency of unbacked MPPs such as star-shaped MPPs [21] located along the axis of an air conditioning duct to dampen its acoustic spinning modes, or micro-perforated cavity floors [22] mounted in a wind-tunnel to reduce the transverse tunnel-cavity modes excited by a shear layer of airflow.

Maa's theory [6,7] showed that a suitable choice of sub-millimetric holes diameter and perforation ratio provides enough acoustic resistance and low acoustic mass reactance to ensure a wide half-absorption bandwidth around the Helmholtz resonance of rigidly-backed MPPs. However, the great bulk of studies on MPPs considers apertures whose characteristic dimension is usually greater than $0.2 \mathrm{~mm}$ and with a low perforation ratio. In order to broaden the bandwidth beyond two octaves, the MPP holes diameters should fall in the range 100-200 $\mu \mathrm{m}$, which would require costly manufacturing processes using machining or laser technologies [12]. A great challenge would be to produce MPPs with hundreds of minute orifices per square centimeters [23]. The photolithographic process, well-suited to produce MEMS (Micro-Electro Mechanical Sensors), has been used in bio-microfluidics to fabricate freestanding micro-perforated membranes made of solid polymer with holes diameter down to $10 \mu \mathrm{m}$ [24]. This technology has been applied to fabricate silicon MPPs with minute holes diameters down to $27 \mu \mathrm{m}$ and a perforation ratio up to 19\%, thereby providing a bandwidth between 3 and 4 octaves with maximum absorption exceeding 0.85 [25]. This complies with an optimisation study of the constitutive parameters of a MPP-MPP-Panel partition [26] that lead to micrometric holes diameter values in order to achieve a "plateau-like" absorption performance greater than 0.9 between $500 \mathrm{~Hz}$ and $2.7 \mathrm{kHz}$.

The goal of the present work is to investigate the absorption, transmission and dissipation properties of micro-capillary plates (MCP) in slip-flow regime characterized by holes diameters between $0.6 \mu \mathrm{m}$ and $50 \mu \mathrm{m}$ as well as a large perforation ratio, typically between 50 and 65\%. The advantage of MCPs is to provide ad-hoc acoustic resistance and, most importantly, very low acoustic reactance over a broad frequency range, which is a key difference with respect to MPPs whose perforation ratio hardly exceed 10-15\%. Since MCPs are compact rigid absorbers with calibrated acoustic resistance that does not vary with frequency, they are potential candidates for the design of low-frequency anechoic terminations. If plugged at the termination of a transmission tube in the no-flow case, they would enable two-port transmission loss measurements through single evaluation of the incident and transmitted sound power in plane wave regime, avoiding the use of the twoload method [27] or without requiring scattering matrix measurement using the two-source method [28]. Also, MCPs calibrated resistance is an advantage with respect to soft porous materials whose resistance may show some variability depending on the mounting boundary conditions [29]. Moreover, the degree of anechoicity of these materials is not always controlled at low frequencies [30] and their shape has to be optimized to avoid prohibitive depth [31]. MCPs could also be used as anechoic wall treatments in open flow ducts as an alternative to perforations covered by a tissue whose resistivity is not well known [30], but this is out of the scope of the current study restricted to the no-flow case.

Dalmont et al. [32] firstly studied in 1989 the input impedance of a coupling cavity backed by a MCP with channel radius $12.5 \mu \mathrm{m}$ in order to achieve a low-frequency anechoic termination of calibrated resistance for closed sound ducts. A suitable MCP resistance value was obtained after minimising the reflection coefficient of the coupling adapter assuming normal incidence and unbacked anechoic load. However, a continuum model [33] was used for sound propagation through the MCP channels in place of a slip-flow model. In the current study, a description of the MCP transfer impedance is proposed in slip- 
flow regime that goes beyond the continuum approach for MCPs with channels radii down to $0.6 \mu \mathrm{m}$. An optimisation strategy is presented based on minimising the acoustic power dissipated by MCPs under normal and oblique incidence plane wave excitations and under general load conditions, including rigid backing. It is examined through modelling and experiments how the MCP optimal resistance depends on the actual load impedance, which is hardly anechoic in practice, and on the plane wave incidence angle, which is relevant for room acoustics applications.

Section 2 describes the morphology of MCPs together with the modelling of sound wave propagation in micrometric channels that exhibit a slip-flow regime in which free-molecular behaviour is accounted for at the channels wall. Section 3 assesses the theoretical absorption predictions against Kundt tube measurements performed on unbacked and backed MCP configurations. Section 4 proceeds with an optimisation study that determines, for a given acoustical load, the optimal MCP impedance that maximizes the dissipated sound power. It shows how to tailor the MCP parameters in order to achieve specific acoustic target performances for a number of loads and incidence angles. Section 5 summarizes the main results and provides future research lines.

\section{Modelling of the MCP acoustical properties}

Sound wave propagation through MCPs with holes diameters typically lower than $10 \mu \mathrm{m}$ exhibit deviations from wellestablished continuum laws [34,35]. This occurs when the holes radius $r_{0}$ approaches, even by several decades, the molecular mean free path $\Lambda$, the ratio $\Lambda / r_{0}$ defining the Knudsen number $K n$. Therefore, two sound wave propagation regimes will be considered that encompass the whole range of MCP pore diameters studied in this work: the continuum regime, embodied in the Navier-Stokes equation and no slip at the walls, valid up to $\mathrm{Kn}=10^{-3}$, and the slip-flow regime, that accounts for tangential slip-velocity boundary condition along the channel walls, first-order accurate in $\mathrm{Kn}$ and valid over the range $10^{-3} \leq \mathrm{Kn} \leq 10^{-1}$. For $10^{-1} \leq \mathrm{Kn} \leq 1$, the continuum assumption breaks down in the bulk of the channel and an early transition regime holds in which higher-order slip wall and extended hydrodynamic equations [36] should be used. For higher Kn, full transition and free-molecular regimes can be numerically modelled by the Lattice Boltzmann Method, but this is out of the scope of this study.

\subsection{MCPs morphology, usage and fabrication}

MCPs are leaded glass plates comprising a large number of identical parallel channels, up to one million per $\mathrm{cm}^{2}$, whose diameter $d_{0}$ ranges from $4 \mu \mathrm{m}$ to $50 \mu \mathrm{m}$ and thickness $t$ from $0.5 \mathrm{~mm}$ to $5 \mathrm{~mm}$ so that the thickness-to-channel diameter ratio, $t / d_{0}$, is typically between 10 and 200. MCPs with electrodes are typically used as image intensifiers or as detectors in cosmic rays and particle physics, but also as photo-multipliers in VLD (Velocimetry Laser Doppler) fluid dynamics instrumentation. Their fabrication uses solid acid-etching process on a glass tube fitted with an array of multi-fibers dissolved in a chemical solution, leaving a glass plate with millions of micro-channels. Nowadays, the cost of small-sized MCPs has been reduced and their fabrication can account for specific values of their constitutive parameters such as the pore diameters, the perforation ratio, the thickness and the geometry (flat or curved, rectangular or disk-shaped, multi-layered).

Fig. 1 shows X-ray laminography pictures of the internal structure of a disk-shaped Glass Capillary Array (GCA) corresponding to GCA I in Table 1 below. One observes in Fig. 1(c and d) that GCA I micro-channels are distributed in a triangular lattice, so that its perforation ratio, defined by $\sigma=2 \pi r_{0}^{2} /\left(\sqrt{3} b^{2}\right)$, reaches $50 \%$ given a pitch (center-to-center channel spacing) $b=33.7 \mu \mathrm{m}$. Moreover, their micro-channels are straight tubes without bias angle and of constant cross-section so that the tortuosity factor is equal to one.
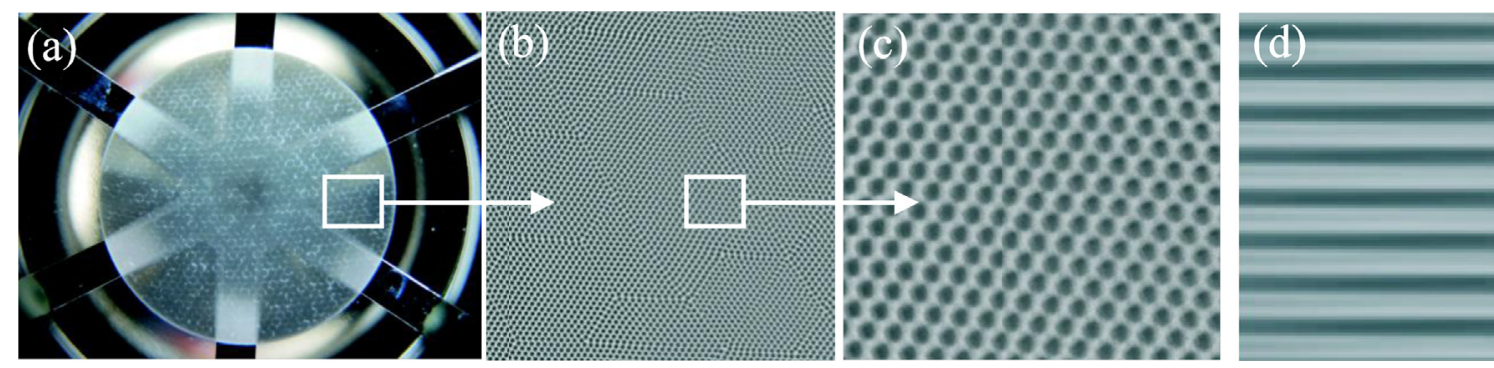

Fig. 1. (a) Photography of GCA I with outer diameter $25 \mathrm{~mm}$; Magnifications of the micro-capillary plate over surfaces of areas $3.6 \mathrm{~mm} \times 3.6 \mathrm{~mm}(\mathrm{~b})$ and $0.7 \mathrm{~mm} \times 0.7 \mathrm{~mm}$ (c): bright and dark zones are respectively linked to the glass and air densities; (d) Cross-sectional imaging of GCA I over a surface of area $0.7 \mathrm{~mm} \times 0.5 \mathrm{~mm}$; (b), (c) and (d) are obtained using X-ray laminography. 
Table 1

Geometrical characteristics of MPP, GCA I, II and III; the aspect ratio is the thickness-to-channel diameter ratio, $t / d_{0}$, with $d_{0}=2 r_{0}$, and the number of channels per $\mathrm{cm}^{2}$ is $10^{-4} \sigma /\left(\pi r_{0}^{2}\right)$ with $r_{0}$ the channels diameter and $\sigma$ the perforation ratio.

\begin{tabular}{|c|c|c|c|c|c|c|}
\hline Plate type & $\begin{array}{l}\text { Channel radius } \\
(\mu \mathrm{m})\end{array}$ & $\begin{array}{l}\text { Knudsen number } \\
\text { Kn }\end{array}$ & $\begin{array}{l}\text { Perforation ratio } \\
(\%)\end{array}$ & $\begin{array}{l}\text { Thickness } \\
(\mu \mathrm{m})\end{array}$ & $\begin{array}{l}\text { Aspect } \\
\text { ratio }\end{array}$ & $\begin{array}{l}\text { Number of channels per } \\
\mathrm{cm}^{2}\end{array}$ \\
\hline MPP & 250 & $1.3 \times 10^{-4}$ & 0.79 & 500 & $1: 1$ & 4 \\
\hline MCP Glass Capillary Array I (GCA I) & 12.5 & $5.1 \times 10^{-3}$ & 50 & 500 & $20: 1$ & $10^{5}$ \\
\hline $\begin{array}{l}\text { MCP Glass Capillary Array II (GCA } \\
\text { II) }\end{array}$ & 5 & $1.3 \times 10^{-2}$ & 68 & 2250 & $225: 1$ & $8.7 \times 10^{5}$ \\
\hline $\begin{array}{l}\text { MCP Glass Capillary Array III (GCA } \\
\text { III) }\end{array}$ & 12.5 & $5.1 \times 10^{-3}$ & 60 & 1000 & 40:1 & $1.2 \times 10^{5}$ \\
\hline
\end{tabular}

\subsection{Sound wave propagation through MCPs in slip-flow regime}

As for GCA I (resp. GCA II and III), the Knudsen numbers in Table 1 correspond to $r_{0}=78 \Lambda$ (resp. $196 \Lambda$ ) with $\Lambda=0.064 \mu \mathrm{m}$ the molecular mean free path for air at normal ambient conditions. Sound wave propagation through these MCPs requires the concept of slip-flows valid for $10^{-3} \leq \mathrm{Kn} \leq 10^{-1}$, i.e. $10 \Lambda \leq r_{0} \leq 1000 \Lambda$. In this regime, continuum laws hold valid in the bulk of the pore, except within thin layers of thickness $\sim \Lambda$ near the pore walls, the so-called Knudsen layers. In these layers, the rate of collisions between the gas molecules and the solid is not high enough to achieve flow momentum and thermodynamic equilibrium, resulting in non-zero velocity slip and temperature jump conditions at the wall [36,37].

Maxwell [38] derived the tangential velocity slip boundary condition in the frame of the kinetic theory of ideal monoatomic gas molecules, assimilated to rigid spheres, in contact with a reflecting wall in isothermal condition. The rationale is applied here to a cylindrical pore of wall surface $\Sigma$, of radius $r_{0}$ and of unit normal $n$ entering the pore. In case of an ideally smooth wall, specular molecular reflection occurs with equal incident and reflected angles. This results in conservation of the molecules tangential momentum and the absence of shear stress exerted on the wall, so that $\partial v /\left.\partial n\right|_{\Sigma}=0$ (perfect slip) with $v$ the tangential mean velocity of the gas molecules and $\partial / \partial n$ the normal derivative with respect to the dimensionless coordinate $r / r_{0}$. In case of a highly rough wall, perfectly diffuse reflection occurs with the random reflection angles being uncorrelated with the incident ones. This results in zero tangential momentum of the reflected molecules and a shear stress transmitted to the wall. Equating the shear stress at the wall and in the fluid leads to the following expression for the slope of the tangential velocity profile at the wall:

$$
\left.\frac{\partial v}{\partial n}\right|_{\Sigma}=\frac{v_{\Sigma}-v_{\text {wall }}}{\left(L / r_{0}\right)}
$$

in terms of $v_{\Sigma}-v_{\text {wall }}$ the slip velocity that reduces, in case of a wall at rest, to $v_{\Sigma}$, the trace of $v$ on the wall, and $L$ the slip distance over which $v$ varies significantly, typically over a length that corresponds to the molecular mean free path $\Lambda$. Hence, Eq. (1) reduces to

$$
v_{\Sigma}=\left.\operatorname{Kn} \frac{\partial v}{\partial n}\right|_{\Sigma} .
$$

In practice, molecular reflection on real walls is essentially diffuse and weakly specular [37], so that a large fraction of the incident tangential momentum is transferred to the wall. This fraction corresponds to $\sigma_{v}$, the tangential momentum accommodation coefficient, with $\sigma_{v}=0$ for perfectly smooth walls and $\sigma_{v}=1$ for extremely rough walls. The Maxwell model can thus be expressed for most practical surfaces through the following slip-flow boundary condition [36-38]

$$
v_{\Sigma}=\left.\frac{2-\sigma_{v}}{\sigma_{v}} \operatorname{Kn} \frac{\partial v}{\partial n}\right|_{\Sigma}=\left.B_{v} \frac{\partial v}{\partial n}\right|_{\Sigma},
$$

that reverts to Eq. (2) for $\sigma_{v}=1$ and that leads to $\partial v /\left.\partial n\right|_{\Sigma}=0$ for $\sigma_{v}=0$.

A similar reasoning has been made by von Smoluchowski [39] and Kennard [40] in the framework of rigid sphere gas from energy flux balance at the wall and in the fluid, to obtain the following temperature jump boundary condition

$$
\tau_{\Sigma}=\left.\frac{2-\sigma_{\tau}}{\sigma_{\tau}} \frac{2 \gamma}{\gamma+1} \frac{\operatorname{Kn}}{\operatorname{Pr}} \frac{\partial \tau}{\partial n}\right|_{\Sigma}=\left.B_{\tau} \frac{\partial \tau}{\partial n}\right|_{\Sigma}
$$

in terms of $\tau_{\Sigma}$ (resp. $\tau$ ) the temperature disturbance at the wall (resp. in the gas), $\gamma=1.402$ the specific heat ratio, Pr $=0.71$ the Prandtl number and $\sigma_{\tau}$ the thermal accommodation coefficient, e.g. the fraction of incident thermal energy flux transferred to the wall. According to Ref. [35], the approximation, $\sigma_{\tau} \approx \sigma_{v}$, is often used. Measurements [36,41] showed that most of the values for $\sigma_{v}$ range between 0.85 and 1.00 for several gas and surface combinations. As for GCAs, $\sigma_{v} \approx 0.9$ has been chosen 
since it corresponds to the tangential momentum accommodation coefficient of the air-glass combination, as reported in Table I of [41].

Assuming straight cylindrical channels with longitudinal axis $z$ undergoing pulsating gas motion induced by an acoustic pressure gradient $\mathrm{d} p / \mathrm{d} z$ in harmonic regime $\left(\mathrm{e}^{\mathrm{j} \omega t}\right)$, the continuum laws such as linearized momentum and energy conservation read [35]

$$
\begin{aligned}
& \frac{1}{r} \frac{\partial}{\partial r}\left[r \frac{\partial v}{\partial r}\right]+k_{v}^{2} v=\frac{1}{\mu} \frac{\mathrm{d} p}{\mathrm{~d} z}, \\
& \frac{1}{r} \frac{\partial}{\partial r}\left[r \frac{\partial \tau}{\partial r}\right]+k_{\tau}^{2} \tau=\frac{k_{\tau}^{2}}{\rho_{0} C_{p}} p
\end{aligned}
$$

where $k_{v}=\sqrt{-\mathrm{j} \omega \rho_{0} / \mu}$ and $k_{\tau}=\sqrt{-\mathrm{j} \omega \rho_{0} C_{p} / \kappa}=k_{v} \sqrt{\operatorname{Pr}}$ are respectively the viscous and thermal diffusion wavenumbers, $C_{p}$ the specific heat capacity under constant pressure, $\kappa$ the thermal conductivity coefficient, $\operatorname{Pr}=\mu C_{p} / \kappa$ the Prandtl number, $\mu$ the air dynamic viscosity and $\rho_{0}$ the air density. Note that, in Eqs. (5) and (6), one assumes that the pressure remains plane, independent of $r$, in the channels.

Given the formal similarity between Eqs. (5) and (6) as well as between the Robin-type boundary conditions, Eqs. (3) and (4), we first focus on the solution to the momentum boundary-value problem (BVP) described by Eqs. (3) and (5) before transposing to the thermal BVP. The general solution to Eq. (5) is the sum of a particular solution and of a general solution without a right-hand side, namely,

$$
v(r, z)=\frac{1}{\mu k_{v}^{2}} \frac{\mathrm{d} p}{\mathrm{~d} z}\left[1-F\left(r ; k_{v}, B_{v}\right)\right]
$$

with $F$ an unknown dimensionless function that is regular over the channel axis at $r=0$. Substituting Eq. (7) into Eqs (3) and (5) leads to the following BVP,

$$
\begin{aligned}
& \frac{1}{r} \frac{\partial}{\partial r}\left[r \frac{\partial F}{\partial r}\right]+k_{v}^{2} F=0 \\
& F_{\Sigma}-\left.B_{v} \frac{\partial F}{\partial n}\right|_{\Sigma}=1
\end{aligned}
$$

whose solution is given by

$$
F\left(r ; k_{v}, B_{v}\right)=\frac{J_{0}\left(k_{v} r\right)}{J_{0}\left(k_{v} r_{0}\right)-B_{v} k_{v} r_{0} J_{1}\left(k_{v} r_{0}\right)},
$$

with $J_{0}$ and $J_{1}$ Bessel functions of the first kind of orders 0 and 1 respectively. Cross-sectional averaging Eq. (7) leads to the mean axial flow rate $\bar{v}$ in the channels defined as $\bar{v}=S_{0}^{-1} \int_{0}^{2 \pi} \int_{0}^{r_{0}} v(r, z) r \mathrm{~d} r \mathrm{~d} \theta$ with $S_{0}=\pi r_{0}^{2}$ the channel cross-sectional area. Given that $\int x J_{0}(x) \mathrm{d} x=x J_{1}(x)$, one gets

$$
\bar{v}=-\frac{1}{\mathrm{j} \omega \rho_{\mathrm{eff}}} \frac{\mathrm{d} p}{\mathrm{~d} z}
$$

with

$$
\rho_{\text {eff. }}=\rho_{0}\left\{1-\frac{2}{k_{v} r_{0}} \frac{J_{1}\left(k_{v} r_{0}\right)}{\left[J_{0}\left(k_{v} r_{0}\right)-B_{v} k_{v} r_{0} J_{1}\left(k_{v} r_{0}\right)\right]}\right\}^{-1},
$$

the effective or dynamic density of the channel. Eq. (11) can be recast as

$$
\frac{\mathrm{d} p}{\mathrm{~d} z}=-Z_{v} \bar{v}
$$

with $Z_{v}=\mathrm{j} \omega \rho_{\text {eff. }}$ the viscous transfer impedance of the channel, given per unit length as follows

$$
Z_{v}=\mathrm{j} \omega \rho_{0} t\left\{1-\frac{2}{k_{v} r_{0}} \frac{J_{1}\left(k_{v} r_{0}\right)}{\left[J_{0}\left(k_{v} r_{0}\right)-B_{v} k_{v} r_{0} J_{1}\left(k_{v} r_{0}\right)\right]}\right\}^{-1} .
$$

The overall transfer impedance of a MCP in the slip-flow regime can then be obtained as $Z_{v} / \sigma$. 
When $\mathrm{Kn} \leq 10^{-3}$, it can be seen from Eq. (3) that $B_{v} \approx 0$. Eq. (14) then reduces to the overall transfer impedance of a MPP in the continuum regime without pore end effects [6]. For MPPs, supplementary added mass and frictional loss effects occur at the holes inlet/outlet. For MCPs, minute tubes radius with respect to the channels length ensures that these end-effects are negligible with respect to the inner effects described by Eq. (14). They are even further reduced due to paramount hole interaction effects, for instance by a factor 8 when $\sigma=50 \%$ [42].

Returning to the thermal BVP given by Eqs. (4) and (6), its solution is formally similar to Eq. (7) and reads

$$
\tau(r, z)=\frac{p}{\rho_{0} C_{p}}\left[1-F\left(r ; k_{\tau}, B_{\tau}\right)\right]
$$

with $F\left(r ; k_{\tau}, B_{\tau}\right)$ obtained from Eq. (10) after substituting $k_{v}$ by $k_{\tau}$ and $B_{v}$ by $B_{\tau}$. Assuming that velocity divergence is mainly longitudinal, the linearized mass conservation equation reads

$$
\rho_{0} \frac{\partial v}{\partial z}=-\mathrm{j} \omega \rho
$$

where the fluid density fluctuation $\rho$ is obtained from the linearized thermodynamic state equation as follows

$$
\rho=\frac{\gamma}{c^{2}} p-\frac{\rho_{0}}{T_{0}} \tau
$$

with $c_{0}$ the sound velocity and $T_{0}$ the unperturbed gas temperature. Substituting Eqs. (15) and (17) into Eq. (16) and noting that $\gamma-1=c^{2} /\left(T_{0} C_{p}\right)$, one gets

$$
\frac{\partial v}{\partial z}=-\mathrm{j} \omega \frac{p}{\rho_{0} c^{2}}\left[1+(\gamma-1) F\left(r ; k_{\tau}, B_{\tau}\right)\right]
$$

Cross-sectional averaging Eq. (18) leads to

$$
\frac{\mathrm{d} \bar{v}}{\mathrm{~d} z}=-Y_{\tau} p
$$

where $Y_{\tau}$ is the thermal transfer admittance of a channel, given per unit length, as follows

$$
Y_{\tau}=\frac{\mathrm{j} \omega}{\gamma p_{0}}\left\{1+(\gamma-1) \frac{2}{k_{\tau} r_{0}} \frac{J_{1}\left(k_{\tau} r_{0}\right)}{\left[J_{0}\left(k_{\tau} r_{0}\right)-B_{\tau} k_{\tau} r_{0} J_{1}\left(k_{\tau} r_{0}\right)\right]}\right\},
$$

with $p_{0}$ the ambient static pressure. The effective or dynamic compressibility, $\chi_{\text {eff. }}$, of a channel in the slip-flow regime, is then defined from Eq. (20) and $Y_{\tau}=\mathrm{j} \omega \chi_{\text {eff. }}$.

In the continuum regime with no-slip boundary conditions, $B_{v}$ and $B_{\tau}$ are zero-valued and the channel effective density and compressibility reduce to the expressions derived by Stinson, respectively associated to Eqs. (48) and (51) in Refs. [43]. Stinson's expressions generalized Zwikker and Kosten approximations [33] over a wide range of frequency and tube radii that are assumed to be greater than $10 \mu \mathrm{m}$, e.g. with $\mathrm{Kn} \leq 6.4 \times 10^{-3}$. Other authors [36,37,44] considered $\mathrm{Kn}=10^{-3}$ as the frontier between the no-slip and the slip-flow regimes and observed it is case-dependent. It is examined in Sec. 2.3 how sensitive are the MCP acoustical properties to the slip-flow regime.

\subsection{MCPs acoustical properties in continuum and slip-flow regimes}

Assuming normal plane wave incidence, the amplitude reflection and transmission coefficients are defined as $r^{\prime}=$ $\left(Z_{1}-Z_{0}\right) /\left(Z_{1}+Z_{0}\right)$ and $t^{\prime}=2 Z_{0} /\left(Z_{1}+Z_{0}\right)$ with $Z_{1}=Z_{v} / \sigma+Z_{0}$, the input impedance of the absorber backed by an anechoic termination, and $Z_{0}=\rho_{0} c_{0}$. In the following, $R_{\mathrm{tr}}$ and $J_{\mathrm{tr}}$ denote the overall transfer resistance and reactance such that $Z_{v} / \sigma=$ $R_{\mathrm{tr}}+\mathrm{j} J_{\mathrm{tr}}$. On can then calculate the dissipation, $\eta=1-\left|r^{\prime}\right|^{2}-\left|t^{\prime}\right|^{2}$, as the fraction of incident energy not reflected, nor transmitted. It is expressed as a function of $\alpha=1-\left|r^{\prime}\right|^{2}$ (resp. $\tau^{\prime}=\left|t^{\prime}\right|^{2}$ ) the energetic absorption (resp. transmission) coefficients. For a MCP backed by a rigid air cavity of depth $D$, the absorption coefficient is obtained from $Z_{1}=Z_{v} / \sigma+$ $j Z_{0} \cot \left(\omega D / c_{0}\right)$.

\subsubsection{Narrow channel approximation to the MCPs slip-flow model}

Narrow channels behaviour occurs if the Shear number $\mathrm{Sh}=r_{0} / \delta_{v}$ is lower than unity, i.e. if the viscous boundary layer thickness, $\delta_{v}=\sqrt{\mu /\left(\omega \rho_{0}\right)}$, exceeds the channels radius $r_{0}$. This occurs for frequencies up to $f_{\max }=\mu /\left(2 \pi \rho_{0} r_{0}^{2}\right)$. It can be seen that the "narrow channel" behaviour is readily met for GCA I, II and III up to $7 \mathrm{kHz}$, the upper frequency range of the experiments presented in Sec. 3. Indeed, $f_{\max }$ equals $94 \mathrm{kHz}$ (resp. $15 \mathrm{kHz}$ ) for GCA I (resp. GCA II and III). Note that a suitable characteristic length scale (CLS) can be defined as $\delta_{v}$ when $\mathrm{Sh}>1$ and as $r_{0}$ when Sh $<1 . r_{0}$ is thus the CLS for GCA I, II and III up 
to $7 \mathrm{kHz}$. If MCPs with larger tubes radius, say $50 \mu \mathrm{m}$, were considered, $f_{\max }$ would be shifted down to $945 \mathrm{~Hz}$ and either CLS should be considered on their specific frequency ranges up to $7 \mathrm{kHz}$.

As MCPs have narrow channel behaviour, $\left|k_{v} r_{0}\right|<1$, over a broad frequency range, we will proceed to a Taylor series expansion of Eqs. (7) and (10) with respect to the Shear number Sh $=\left|k_{v} r_{0}\right|$ up to order four. This leads to the following axial velocity profile in slip-flow regime,

$$
v(r, z)=\frac{1}{\mu k_{v}^{2}} \frac{\mathrm{d} p}{\mathrm{~d} z}\left[\mathrm{j} \beta_{v}-\frac{k_{v}^{2}\left(r_{0}^{2}-r^{2}\right)}{4}\left(1-\beta_{v}\right)\right],
$$

with $\beta_{v}=\left(B_{v} / 2\right)\left(k_{v} r_{0}\right)^{2}$. The first term is the slip velocity and the second term is a parabolic velocity profile. When $B_{v}=0$, Eq. (21) reduces to the well-known Poiseuille velocity profile, $v(r, z)=-(\mathrm{d} p / \mathrm{d} z)\left(r_{0}^{2}-r^{2}\right) /(4 \mu)$, valid for narrow channels in continuum regime.

Performing a similar series expansion in Eqs. (14) and (20) leads to

$$
\begin{aligned}
& Z_{v} \approx \frac{1}{1-4 B_{v}}\left\{\frac{8 t \mu}{r_{0}^{2}}+\mathrm{j} \frac{4}{3} \omega \rho_{0} t\left(1-6 B_{v}\right)\right\}, \\
& Y_{\tau} \approx \frac{\mathrm{j} \omega}{p_{0}}\left\{1-\mathrm{j} \frac{(\gamma-1)}{8 \gamma} \frac{C_{p}}{\kappa} \omega \rho_{0} r_{0}^{2}\left(1-4 B_{\tau}\right)\right\},
\end{aligned}
$$

which reduce, when $B_{v}=B_{\tau}=0$, to Zwicker and Kosten low frequency approximation for the transfer impedance and admittance of narrow channels in continuum regime [33]. One observes from Eq. (23) that, for MCPs with radius in the range $2-25 \mu \mathrm{m}$ and at low frequencies, $\operatorname{Real}\left(Y_{\tau}\right) \approx 0$, so that there is no thermal dissipation in capillary channels. Moreover, the air particle motion is isothermal at low frequencies. The axial gradient of average intensity, $\mathrm{d} I / \mathrm{d} z=-0.5 \mathrm{Real}\left(Y_{\tau}\right)|p|^{2}-$ $0.5 \operatorname{Real}\left(Z_{v}\right)|\bar{v}|^{2}$, thus reduces to $\mathrm{d} I / \mathrm{d} z \approx-4 t \mu|\bar{v}|^{2}\left\{r_{0}^{2}\left(1-4 B_{v}\right)\right\}^{-1}$ and viscous effects dominate the dissipation of acoustical energy.

Fig. 2 shows the Knudsen number dependency of $\alpha, \tau^{\prime}$ and $\eta$ for a $0.5 \mathrm{~mm}$ thick micro-perforate with a high density of holes $\left(\sigma=50 \%, b \approx 1.35 d_{0}\right)$ with $r_{0}$ decreased from $635 \mu \mathrm{m}$ down to $0.64 \mu \mathrm{m}(10 \Lambda)$ while Kn increases accordingly. It can be seen that the narrow channel approximation given by Eq. (22) well approximates the exact expression given by Eq. (14) over a broad range of Knudsen numbers encompassing the continuum regime.

In this regime $\left(\mathrm{Kn} \leq 10^{-3}\right)$, almost all of the incident energy is transmitted by the highly porous MPP and is weakly dissipated. As the holes radius decreases, the transmission leakage decreases while back-reflexion increases, but not at the same rate, so that part of the incident energy is dissipated within the MCP. A maximum of dissipation occurs in slip-flow regime that reaches $\eta_{\max }=0.5$ for an optimal value of the holes radius $r_{0}^{\text {opt }}=12.8 \mu \mathrm{m}\left(\mathrm{Kn}=5 \times 10^{-3}\right)$. It will be shown in Sec. 3 that $\eta$ cannot exceed 0.5 for a single unbacked MCP under normal incidence. One also observes from Table 1 and Fig. 2 that GCA I $\left(\mathrm{Kn}=5.1 \times 10^{-3}\right)$ is close to the optimal configuration.

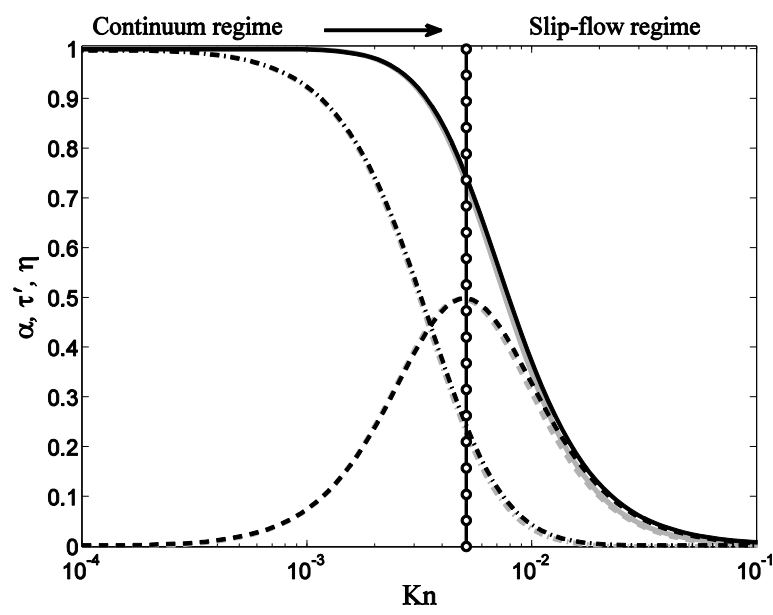

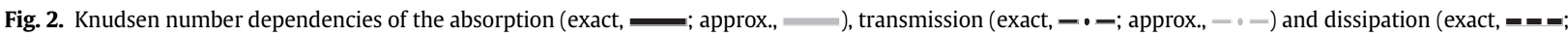
approx, $=--)$ ) coefficients of a MCP $(t=0.5 \mathrm{~mm}, \sigma=50 \%$ ) assuming a slip-flow regime at $500 \mathrm{~Hz}$; the vertical line with circles (-o-O) corresponds to GCA I. 


\subsubsection{Comparison between MPP and MCPs acoustical properties}

From the narrow channel approximation given by Eq. (22) for the viscous transfer impedance of a micro-channel, one finds that the overall MCP transfer impedance, $Z_{v} / \sigma$, reduces to a pure resistance given by,

$$
R_{\mathrm{tr}} \approx \frac{8 t \mu}{\sigma r_{0}^{2}\left(1-4 B_{v}\right)}
$$

It follows an inverse-power law of order 4 with respect to the channels radius and does not depend on frequency, which $a$ priori makes MCPs suitable calibrated acoustic resistances. Still from Eq. (22), the magnitude of the specific reactance, $\left|J_{\operatorname{tr}} / Z_{0}\right| \approx 4 \omega t /\left(3 \sigma c_{0}\right)$, is found to be almost zero-valued over a broad frequency range due to the MCPs high perforation ratio. These are very distinct features from the MPPs impedance properties.

Fig. 3 illustrates these differences, both in the unbacked and backed configurations. When unbacked [Fig. 3(a)], the MCPs exhibit flat absorption over the whole frequency bandwidth, that reaches $\alpha=0.72$ for GCA I, whereas the MPP absorption falls above $200 \mathrm{~Hz}$ due to increasing back-reflections. When backed by a $5 \mathrm{~cm}$ depth cavity [Fig. 3(b)], the MPP provides narrowband absorption whose half-bandwith extends over one octave around the Helmholtz resonance with $\alpha_{\text {max }}=0.88$ at $f_{\mathrm{H}}=629 \mathrm{~Hz}$. GCA I reaches similar maximum value $\alpha_{\max }=0.85$, but provides much broader absorption with a halfbandwidth that spans almost 9 octaves around $f_{\mathrm{H}}=1668 \mathrm{~Hz}$.

Note that $f_{\mathrm{H}}$ is increased up to $1668 \mathrm{~Hz}$ due to a lower effective mass per unit area of GCA I with respect to the MPP. This is reflected in Fig. 3(d) which shows that, unlike the MPP, the MCPs exhibit minute specific reactance over a wide frequency band. Fig. 3(c) confirms that MCPs act as constant acoustic resistances constant whose value is mainly determined by their micro-channels radius. It can be seen that GCA I with $12.5 \mu \mathrm{m}$ channels radius has an input resistance of about $3.3 \mathrm{Z}_{0}$, which is close to $3 Z_{0}$, the optimal input impedance derived in Sec. 3. However, GCA II presents a too high input resistance (46 $\left.\mathrm{Z}_{0}\right)$ due

(a)

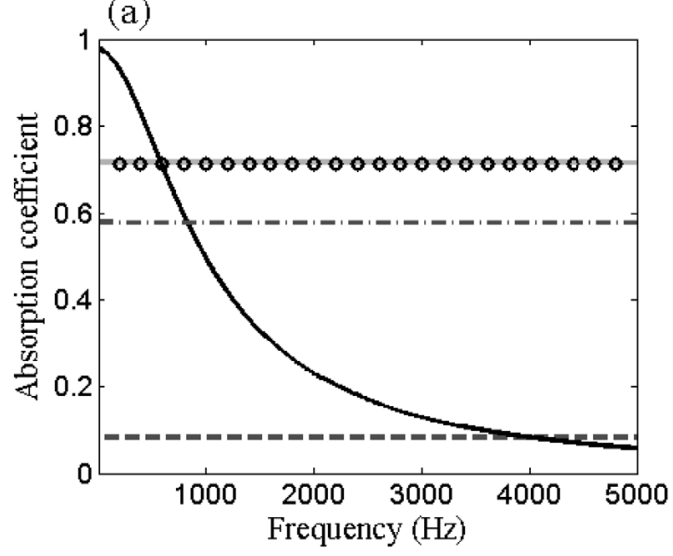

(c)

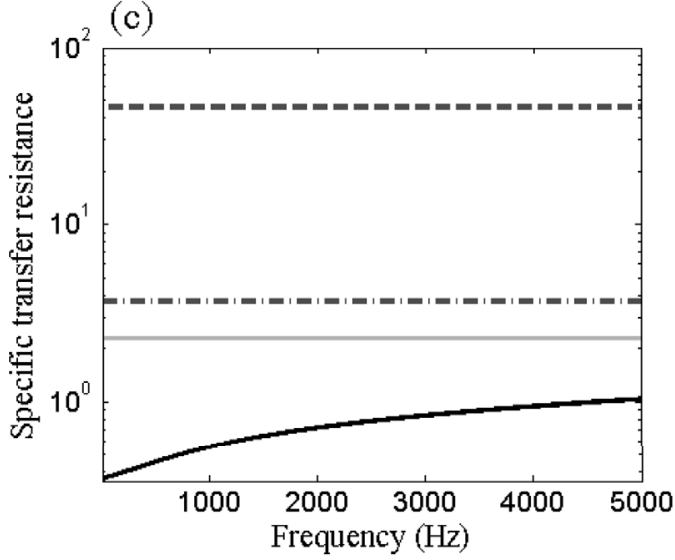

(b)

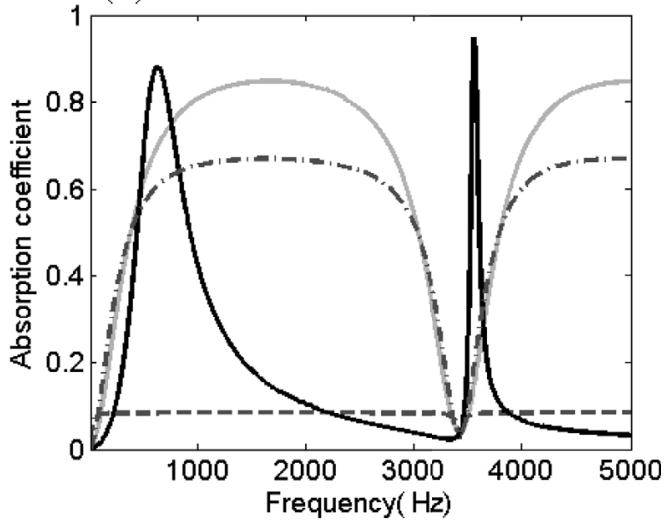

(d)

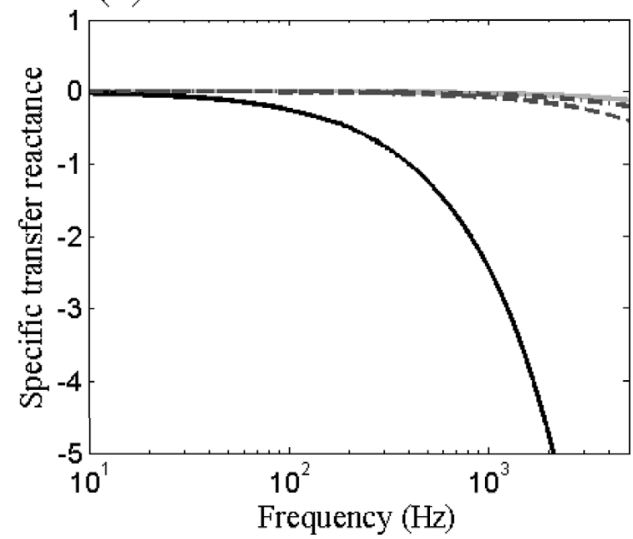

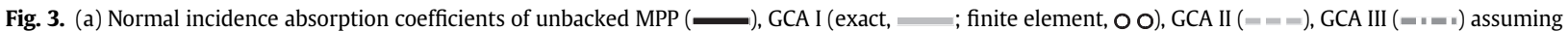
anechoic load conditions; (b) Normal incidence absorption coefficients of rigidly-backed MPP ( depth $D=0.05 \mathrm{~m}$; (c) Frequency dependence of the specific transfer resistance, $R_{\mathrm{tr}} / Z_{0}$, related to MPP $(\square)$, GCA I (

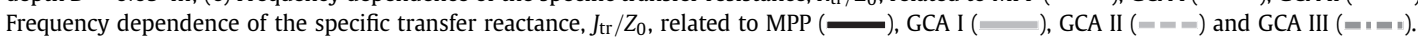


to extremely small channels radius $(5 \mu \mathrm{m})$ and responsible for its poor absorption performance in unbacked and backed configurations.

\subsubsection{Discussion on the validity of the continuum model for MCPs in slip-flow regime}

The theoretical predictions using the slip-flow model have been assessed against Finite Element Method (FEM) using the Thermoviscous Acoustics model implemented in Comsol Multiphysics in the Frequency Domain. This continuum model assumes no-slip velocity and isothermal conditions at the channel walls. The maximum element size was set to $r_{0} / 5$ and 4 layers of thickness $r_{0} / 20=\min _{f \leq 7 \mathrm{kHz}}\left(r_{0} / 20,0.3 \delta_{v}\right)$ constituted the boundary layer mesh to ensure a constant mesh thickness up to $7 \mathrm{kHz}$. It can be seen from Fig. 3(a) that a good agreement is observed between the theoretical and FEM models of GCA I absorption properties. Fig. 4(a) shows the axial gradient of acoustic pressures through the channels of GCA I at $500 \mathrm{~Hz}$. At this frequency, $\delta_{v} \approx 5.5 r_{0}$ and a magnified view in Fig. 4(b) would show a Poiseuille-type velocity profile in the channels crosssection. Fig. 4(c) confirms isothermal conditions within the fluid domains occupied by the MCP channels.

Although the continuum approach is not valid in slip-flow regime, it is found to be predictive of the MCP acoustical properties as long as $\mathrm{Sh}<1$. Indeed, assuming narrow channel behaviour $(\mathrm{Sh}<1)$, one gets from Eq. (14), $Z_{v}^{\mathrm{SF}} \approx$ $\mathrm{j} \omega \rho_{0} t\left\{1-\left(1+0.6 \mathrm{j} \mathrm{Kn} \mathrm{Sh}^{2} Q(\operatorname{Sh} \sqrt{-\mathrm{j}})\right) Q(\operatorname{Sh} \sqrt{-\mathrm{j}})\right\}^{-1}$ in slip-flow regime, with $Q(x)=2 J_{1}(x) /\left[x J_{0}(x)\right]$. It differs from $Z_{v}^{\mathrm{C}}=\mathrm{j} \omega \rho_{0} t\{1-Q(\operatorname{Sh} \sqrt{-\mathrm{j}})\}^{-1}$ in continuum regime by a factor $\mathrm{Kn} \mathrm{Sh}^{2} \mathrm{Q}(\mathrm{Sh} \sqrt{-\mathrm{j}})$ which is much smaller than unity in continuum $(\mathrm{Kn}<<1)$ or in slip-flow $(\mathrm{Sh}<<1)$ regimes since $Q(\mathrm{Sh} \sqrt{-\mathrm{j}}) \approx 1-\mathrm{jSh}{ }^{2} / 8+O\left(\mathrm{Sh}^{4}\right)$ when $\mathrm{Sh}<1$. In case of extremely small channels in slip-flow regime, e.g. when Kn tends towards 0.1, it is of interest to accurately determine how much $Z_{v}^{\mathrm{SF}}$ differs from $Z_{v}^{\mathrm{C}}$. Inserting the second-order series expansion of $Q$ in the above slip-flow and continuum models, one gets $Z_{v}^{\mathrm{SF}} \approx Z_{v}^{\mathrm{C}}\left\{1+4 \mathrm{Kn}\left(2-\sigma_{v}\right) / \sigma_{v}\right\}^{-1}$, or equivalently $Z_{v}^{\mathrm{C}} / Z_{v}^{\mathrm{SF}} \approx 1+4.9 \mathrm{Kn}$, with $Z_{v}^{\mathrm{C}} \approx 8 \omega \rho_{0} t / \mathrm{Sh}^{2}$. It shows that $Z_{v}^{\mathrm{C}}$ increasingly overestimates $Z_{v}^{\mathrm{SF}}$ as $\mathrm{Kn}$ tends towards 0.1. This is in accordance with Fig. 5(a) that predicts a relative error between $Z_{\nu}^{\mathrm{C}}$ and $Z_{v}^{\mathrm{SF}}$ reaching $49 \%$ at $\mathrm{Kn}=0.1$. Note that this discrepancy occurs between very high resistance values $\left(Z_{v}^{\mathrm{C}} \approx 424 Z_{0}\right.$ and $\left.Z_{v}^{\mathrm{SF}} \approx 285 Z_{0}\right)$ so that it hardly affects the corresponding low values of the normal incidence absorption coefficients, $\alpha^{\mathrm{C}}$ and $\alpha^{\mathrm{SF}}$, as well as the minute transmission and dissipation coefficients, as seen from Fig. 5(b).

An overall picture is obtained when examining the narrow channel approximation of the ratio, $r^{\mathrm{C}} / r^{\mathrm{SF}}$, between the reflection coefficients in continuum and slip-flow regimes under a general incidence angle $\theta . Z_{\nu}$ is then replaced by the effective impedance $Z_{\nu} \cos (\theta)$ and one gets $r^{\mathrm{C}} / r^{\mathrm{SF}} \approx 1+4 \mathrm{Kn}\left(2 / \sigma_{v}-1\right)\left\{1+4 \mu t \cos (\theta) /\left(Z_{0} r_{0}^{2}\right)\right\}^{-1}$. For extremely small holes and for normal incidence $\left(\theta=0^{\circ}\right)$, the term $4 \mu t /\left(Z_{0} r_{0}^{2}\right)$ is dominant so that $r^{\mathrm{C}} / r^{\mathrm{SF}} \approx 1$ and $\alpha^{\mathrm{C}} \approx \alpha^{\mathrm{SF}}$. However, when $\theta$ tends towards $90^{\circ}$, the term $4 \mu t \cos (\theta) /\left(Z_{0} r_{0}^{2}\right)$ tends to zero and $r^{\mathrm{C}} / r^{\mathrm{SF}}$ increases up to 1.49 . Hence, under almost grazing incidence $\left(\theta>80^{\circ}\right)$, the continuum model underestimates the absorption coefficient in slip-flow regime by up to $31 \%$, the trend being

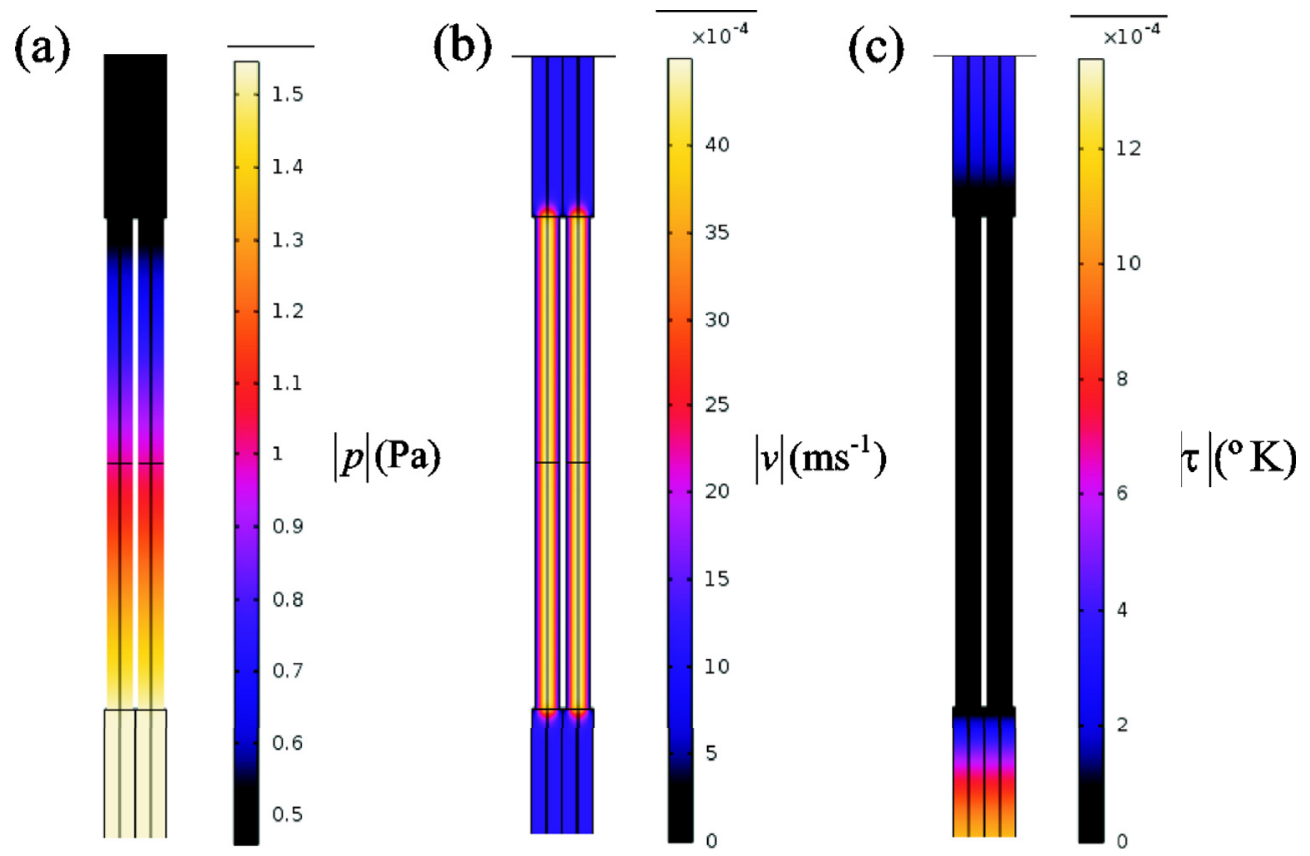

Fig. 4. Spatial distribution of the magnitude of (a) acoustic pressure, (b) acoustic velocity and (c) temperature disturbance calculated by FEM in two GCA I channels whose front side at the bottom of the figure undergoes an incident plane wave at $500 \mathrm{~Hz}$. 


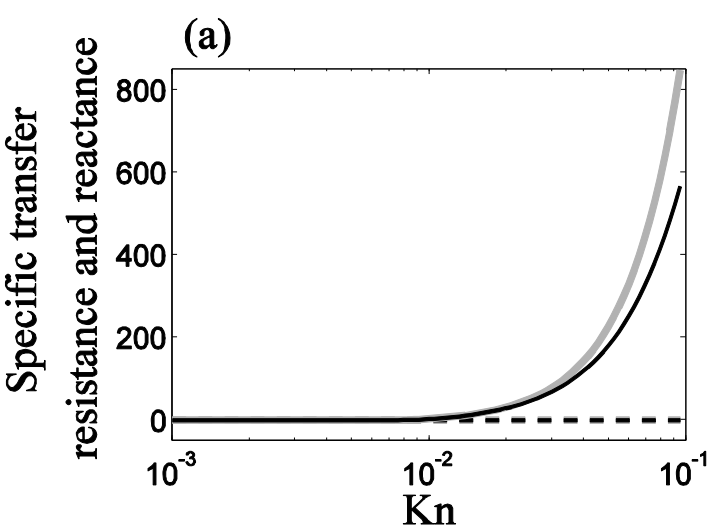

(c)

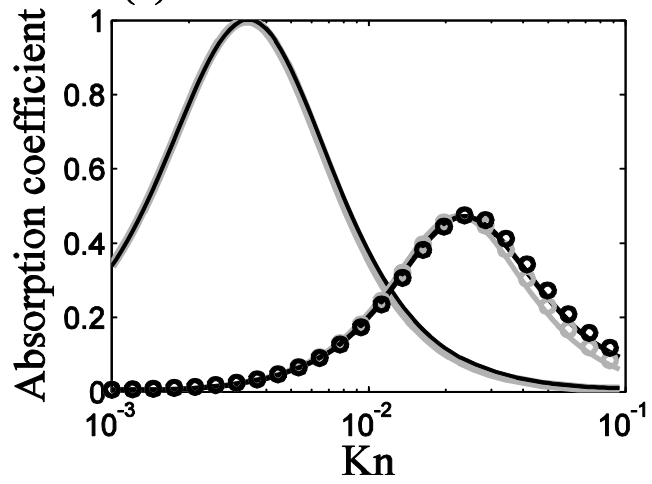

(b)

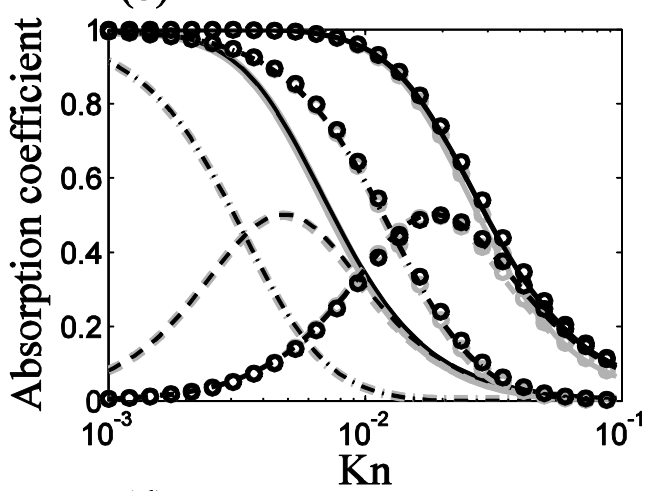

(d)

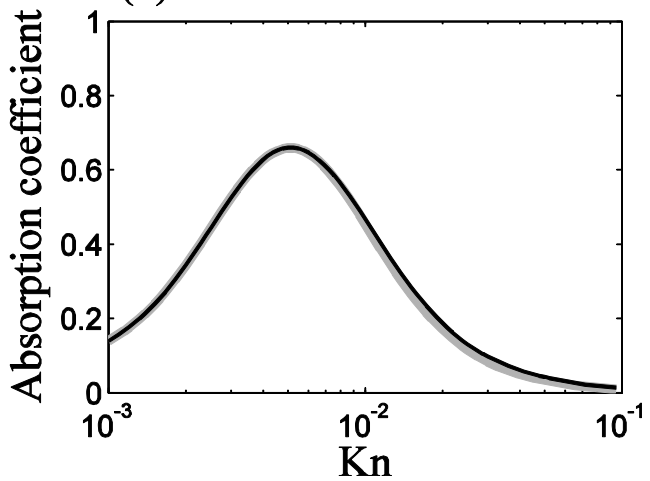

Fig. 5. Knudsen number dependency of the acoustical properties of a MCP ( $t=0.5 \mathrm{~mm}, \sigma=50 \%$ calculated at $5 \mathrm{kHz}$ from the slip-flow (black, $\left.Z_{\nu}^{\mathrm{SF}}\right)$ and continuum (grey, $Z_{v}^{\mathrm{C}}$ ) exact models: (a) Specific overall transfer resistance (solid) and reactance (dashed) of the MCP; (b) Normal (lines, $\theta=00^{\circ}$ ) and almost grazing (lines with circles, $\theta=86^{\circ}$ ) incidence absorption (solid), transmission (dash-dotted) and dissipation (dashed) coefficients of the unbacked MCP assuming anechoic load condition; (c) Normal (lines, $\theta=0^{\circ}$ ) and almost grazing (lines with circles, $\theta=86^{\circ}$ ) incidence absorption coefficient of the MCP rigidly-backed by a cavity of depth $D=0.05 \mathrm{~m}$; (d) Random diffuse field absorption coefficient of the MCP rigidly-backed by a cavity of depth $D=0.05 \mathrm{~m}$.

observed in Fig. 5(b). This also occurs for the transmission and dissipation coefficients. A similar conclusion holds for the absorption coefficient of rigidly-backed MCPs under plane wave incidence [Fig. 5(c)].

Fig. 5(b) and (c) show that $\alpha^{\mathrm{C}}$ underestimates $\alpha^{\mathrm{SF}}$ under almost grazing incidence for Kn $>0.03$, e.g. for channels radius $r_{0}<$ $2 \mu \mathrm{m}$. However, as seen from Fig. 5(d), the continuum and slip-flow models hardly affect the absorption coefficient of rigidlybacked MCPs under a diffuse field random excitation defined as $2 \int_{0}^{\pi / 2} \alpha(\theta) \cos (\theta) \sin (\theta) \mathrm{d} \theta$. The absorption is then weighted by a factor $\cos (\theta)$ that tends to be small near grazing angles. In summary, the slip-flow model should be used instead of the continuum model to predict the transfer impedance of MCPs with channels radii lower than $2 \mu \mathrm{m}(\mathrm{Kn}>0.03)$ as well as their absorption coefficient under near-grazing incident excitations $\left(\theta>80^{\circ}\right)$. Otherwise, both approaches provide similar results.

\section{Experimental study}

To verify the accuracy of the proposed models, measurements have been performed to determine the absorption coefficient of several MCP absorbers using a small impedance tube installed vertically in a semi-anechoic environment, as shown in Fig. 6(a). The procedure is based on the two-microphone method [45].

A loudspeaker, connected at the base of the tube, generates a random pressure field that propagates towards the MCP holder. The tube made of steel is $1 \mathrm{~cm}$-thick, $80 \mathrm{~cm}$-long and has an inner radius $R=1.5 \mathrm{~cm}$ that provides a maximum frequency of analysis slightly less than $6700 \mathrm{~Hz}$, the first duct cut-on frequency, so that $k_{0} R \leq 1.84$ with $k_{0}$ the acoustic wavenumber. The transfer function $H_{12}$ is estimated between the acoustic pressures $p_{1}$ and $p_{2}$ measured at the positions of two $1 / 4^{\prime \prime}$ condenser microphones located upstream of the sample and separated by a distance $d=5 \mathrm{~cm}$. Such spacing stays lower than the smallest half-wavelength at the duct cut-on frequency and provides a lower frequency limit of $200 \mathrm{~Hz}$ above which results are known to be accurate. The reflection coefficient at the holder position, $z=0$, takes the form, $r_{h}^{\prime}(z=0)=$ $\mathrm{e}^{-2 \mathrm{j} k_{0}(d+e)}\left(H_{12}-\mathrm{e}^{-\mathrm{j} k_{0} d}\right) /\left(\mathrm{e}^{\mathrm{j} k_{0} d}-H_{12}\right)$, with $e=6.7 \mathrm{~cm}$ the distance between microphone 1 and the holder.

Because the MCP has a radius of $1.275 \mathrm{~cm}$ that is smaller than the duct inner radius, it requires a thick PVC adaptor inserted into the tube and onto which the MCP is fixed, as shown in Fig. 6(b). The adaptor introduces a constriction of radius $r_{c}=1 \mathrm{~cm}$ 

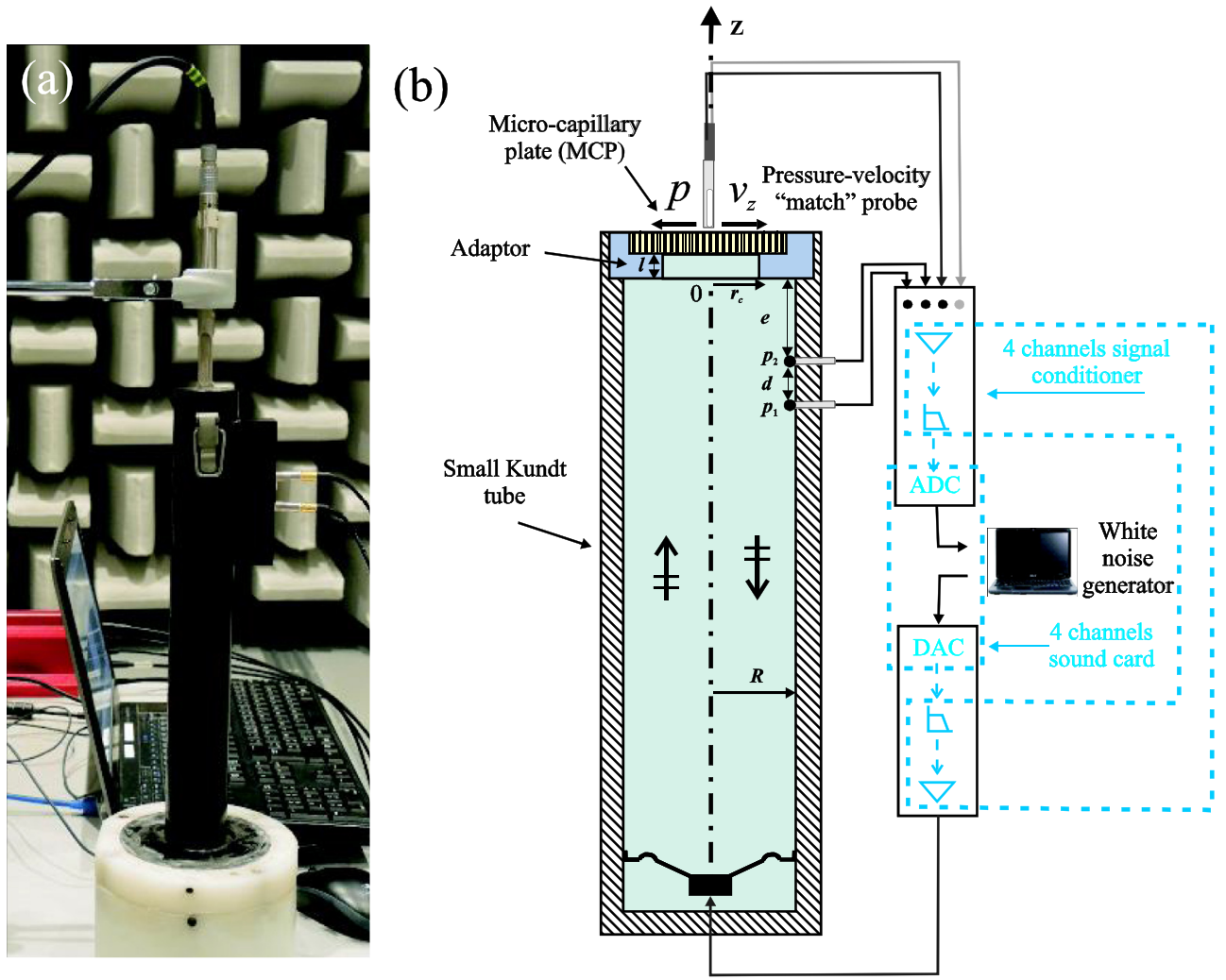

Fig. 6. (a) Photography of the small Kundt tube used to measure the absorption coefficient of an unbacked MCP whose acoustic radiation in a semi-anechoic facility is characterized by a pressure-velocity probe; (b) Schematic of the whole experimental set-up with cross-sectional view of the PVC circular adaptor into which the MCP was set.

and thickness $l=1 \mathrm{~cm}$. Applying continuity of the pressures and acoustic flow rate at $z=0$ leads to the following estimate of the MCP specific input impedance at $z=l$,

$$
\frac{Z_{1}}{Z_{0}} \approx \frac{r_{c}^{2}\left(1+r_{h}^{\prime}\right) \cos \left(\tilde{k}_{0, r} l\right)-\mathrm{j} R^{2}\left(1-r_{h}^{\prime}\right) \sin \left(\tilde{k}_{0, r} l\right)}{R^{2}\left(1-r_{h}^{\prime}\right) \cos \left(\tilde{k}_{0, r} l\right)-\mathrm{j} r_{c}^{2}\left(1+r_{h}^{\prime}\right) \sin \left(\tilde{k}_{0, r} l\right)},
$$

with $\tilde{k}_{0, r}=-\mathrm{j} \sqrt{Z_{v, r} Y_{\tau, r}}$ a complex wavenumber that accounts for viscothermal attenuation at the adaptor walls, $r_{0}$ being replaced by $r_{c}$ in Eqs. (14) and (20) to get $Z_{v, r}$ and $Y_{\tau, r}$ in continuum regime. The absorption coefficient is then readily obtained from Eq. (25). Note that, during the experiments, the output signals from the sensors were acquired using the OROS (type OR38) multi-channel system over a bandwidth $80 \mathrm{~Hz}-6.7 \mathrm{kHz}$, at a sampling rate of $12.8 \mathrm{kHz}$ and with a spectral resolution of $1.56 \mathrm{~Hz}$, triggered on the generation of a white noise drive signal.

Fig. 7(a) shows that plugging the thin GCA I disk onto the open tube termination, via the adaptor, significantly enhances the absorption coefficient over a broad bandwidth, as it reaches a constant value of 0.86 up to $k_{0} R=0.3$ and then slowly decreases down to 0.7 up to $k_{0} R=1.84$. This is appreciated when compared to the fraction of incident energy not reflected by the open adaptor which is minute below $k_{0} R=0.4$ and monotonically reaches a value of 0.65 towards $k_{0} R=1.84$. This open tube behaviour is well predicted by the $\left(\nu, \alpha^{\prime}\right)$ model for the reflection coefficient of unflanged cylindrical ducts [46] given by $r_{\text {open }}^{\prime}(\omega)=-\left(1+\mathrm{j} k_{0} R / \alpha^{\prime}\right)^{-(\nu+1)}$ with $\alpha^{\prime}=1.2266$ and $\nu=0.504$. One also observes in Fig. 7(a) that the theoretical model, which assumes plane wave anechoic load, provides a flat absorption spectrum that underpredicts the measured absorption values by $20 \%$ up to $k_{0} R=0.3$. The load radiation impedance of the unbacked MCPs has thus been characterized.

Near-field collocated measurements have been performed of both the pressure and the air particle normal velocity radiated by the MCPs in free-field using a miniature $0.005 \mathrm{~m}$ pressure-velocity $(p-v)$ probe. A surface-averaged estimate of GCA I radiation impedance has been obtained from a set of $p-v$ measurements performed over a circular surface located at a fixed stand-off distance, $d_{S_{-O}}=0.01 \mathrm{~m}$, from the MCP radiating side. The measurement points are distributed over four diametral lines located at $45^{\circ}$ from each other, each line comprising 21 points separated by $1.5 \mathrm{~mm}$. The short distance $d_{S_{-}-}$ avoids the presence of pressure nodes at the microphone over the measurement bandwidth up to $f_{\max }=6.7 \mathrm{kHz}$ since $d_{S_{-} \mathrm{O}}<$ 
(a)

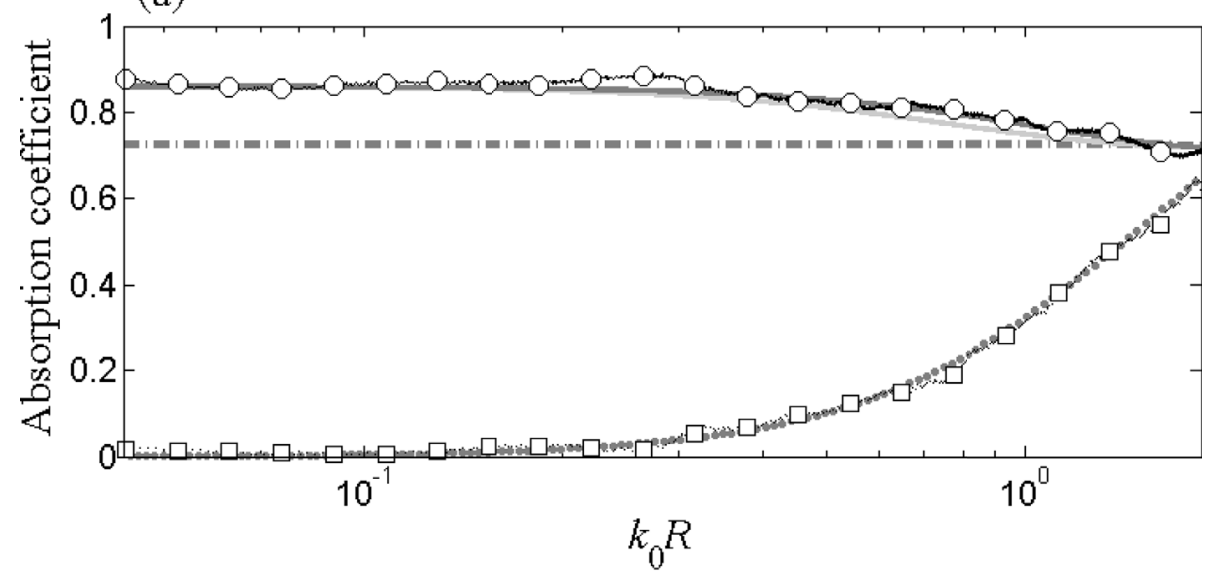

(b)

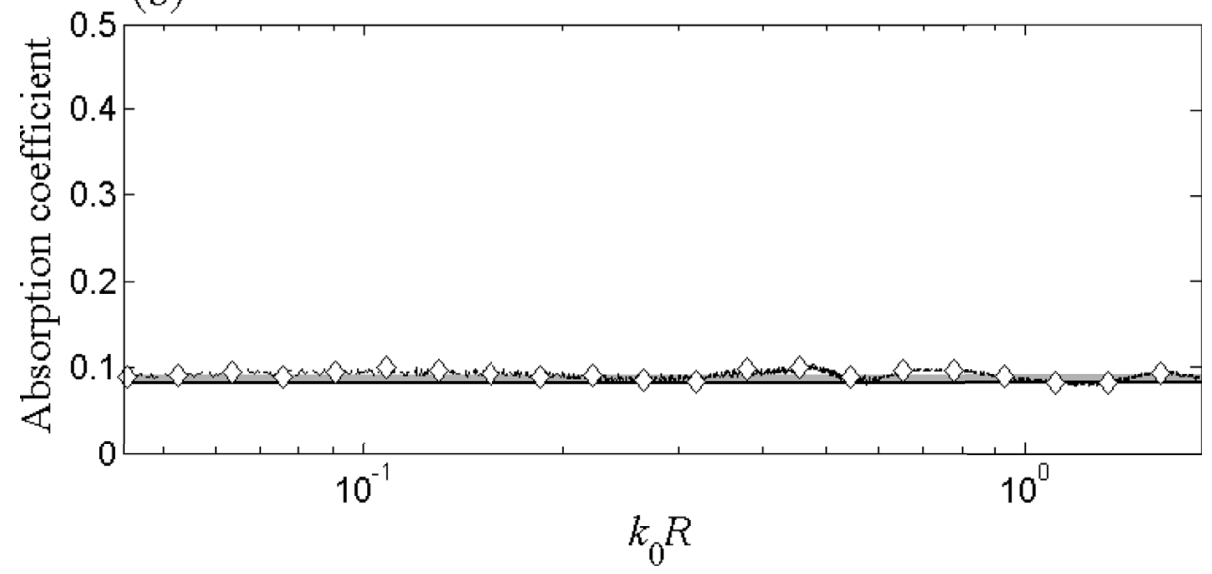

Fig. 7. (a) Normal incidence absorption coefficients of unbacked GCA I: measured (-○-) and predicted assuming unflanged (

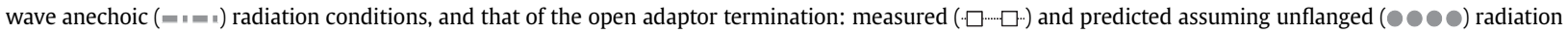
condition; (b) Normal incidence absorption coefficient of unbacked GCA II: measured ( $\diamond \diamond)$ and predicted assuming plane wave anechoic radiation condition (continuum regime, $\longrightarrow$; slip-flow regime, $\longrightarrow$ ).

$c_{0} /\left(4 f_{\max }\right)$. It also keeps the signal-to-noise ratio above $20 \mathrm{~dB}$ at both the microphone and the particle velocity sensors. Prior to the experiments, the amplitude and phase responses of the $p-v$ sensors were calibrated in a standing wave tube [47] and in anechoic conditions.

It can be seen in Fig. 8 that the load impedance measured behind GCA I is obviously different from a plane wave anechoic condition. Because the MCP is highly porous, its load impedance does not substantially differ from that of an open cylindrical termination, as hypothesized in Ref. [32]. A good agreement is observed between the measured surface-averaged radiation impedance and the plane wave radiation impedance predicted from Zorumski's infinite flanged model [48] and corrected by spatial variation effects induced by higher-order evanescent axisymmetrical modes [46]. It was observed that these higherorder modes tend to lower the plane wave mode radiation resistance and reactance values below $k_{0} R \approx 1.84$ and improve the correlation with Zorumski's model. The measured radiation impedance also well correlates, especially below $k_{0} R \approx 0.7$, with the $\left(\nu, \alpha^{\prime}\right)$ model proposed in Ref. [46] for flanged cylindrical ducts with $\alpha^{\prime}=0.8216$ and $\nu=0.350$, but not so well for the unflanged model that does not account for the effects of the higher order modes, nor of the finite thickness flange.

Nevertheless, Fig. 7(a) shows that accounting for both the radiation models in GCA I load impedance significantly improves the correlation between the predicted and measured absorption coefficients. Because GCA II has a very high transfer resistance as well as a small transfer reactance, the theory predicts a low flat absorption spectrum of about 0.1 , which is confirmed by the measurements, as it can be seen from Fig. 7(b). Note that GCA II absorption values are insensitive to the load impedance. Moreover, given GCA II Knudsen number $\left(\mathrm{Kn}=1.3 \times 10^{-2}\right)$, the slip-flow theory slightly improves the agreement with the measurements when compared to the continuum theory whereas both theories lead to hardly distinguishable results for GCA I $\left(\mathrm{Kn}=5.1 \times 10^{-3}\right)$. 
(a)

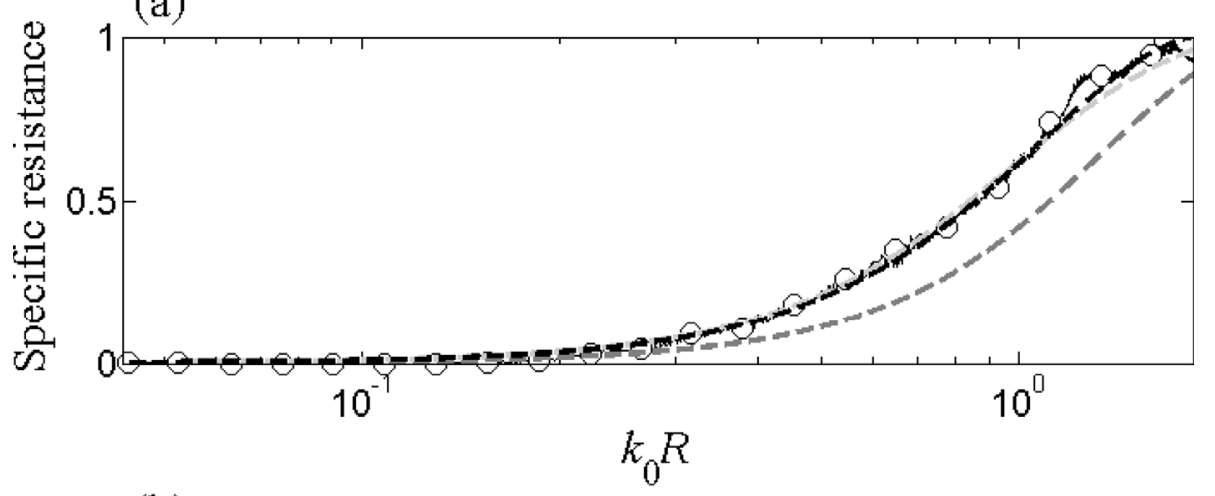

(b)

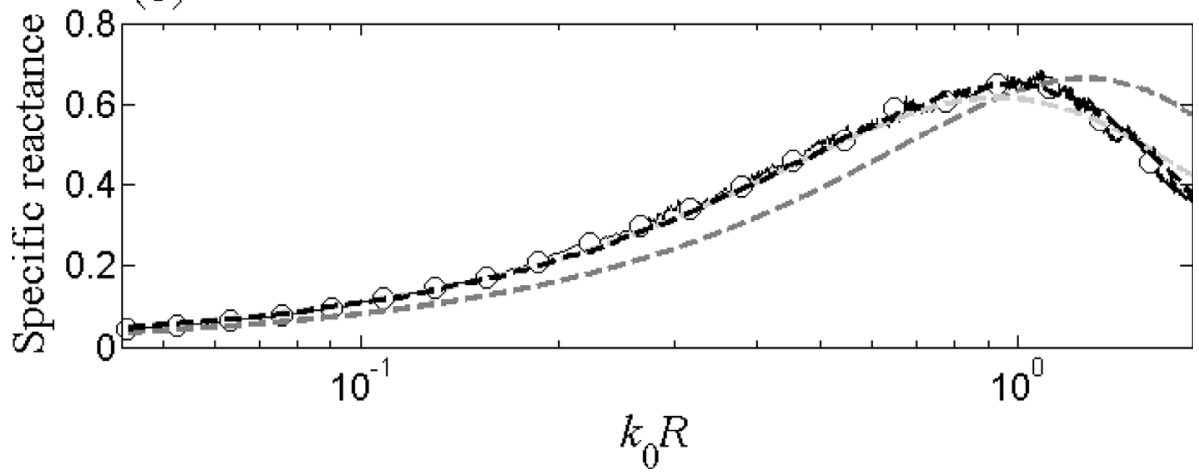

Fig. 8. Specific radiation resistance (a) and reactance (b) of GCA I when evaluated from surface-averaged $p$ - $v$ free-field measurements (-o-a-), from Zorumski's radiation impedance model (- - - ) accounting for the effects of six higher order radial modes and from the approximate $\left(\nu, \alpha^{\prime}\right)$ radiation impedance models assuming flanged $(-=-)$ and unflanged $(---)$ terminations.

Theory and experiments showed that MCP terminations radiating in free-field are able to provide, in the plane wave domain, wideband flat absorption performance that can be monitored by varying the channels radius. Of interest is to examine the absorption performance of MCPs backed by opened and closed cavities.

The absorption measurements shown in Fig. 9(a) on GCA I rigidly-backed by a $24 \mathrm{~mm}$-depth cavity confirm the practical ability of MCPs to provide a broad half-absorption bandwidth that here spans almost 12 octaves around $f_{\mathrm{H}}=3320 \mathrm{~Hz}$ with $\alpha_{\max }=0.86$. The measured absorption curve reasonably agrees with the model that considers a MCP input impedance of the form, $Z_{1}=Z_{v} / \sigma+\mathrm{j} Z_{0}\left(r_{c}^{2} / R^{2}\right) \cot \left(\tilde{k}_{0, R} D\right)$, with $Z_{v} / \sigma$ given by Eq. (24) and $\tilde{k}_{0, R}=-\mathrm{j} \sqrt{Z_{v, R} Y_{\tau, R}}$, a complex wavenumber that accounts for viscothermal attenuation at the cavity walls. It is noteworthy that the absorption can be further enhanced by fine tuning of the channels radius to achieve a critical coupling condition [49] for which the viscous losses in the capillaries balance the leakage due to back-reflection. As shown in Fig. 9(a), this is obtained when enlarging the channels radius from $12.5 \mu \mathrm{m}$ to $17.5 \mu \mathrm{m}$, thereby providing an absorption coefficient greater than 0.99 between $2.8 \mathrm{kHz}$ and $3.8 \mathrm{kHz}$, albeit with a somewhat reduced half-bandwith with respect to GCA I.

The drawback of this resonant absorber is its poor absorption performance at low- and mid-frequencies, here below $1 \mathrm{kHz}$. As illustrated in Fig. 10, a hybridized MCP made up of a parallel arrangement of backed and unbacked MCPs with equal surface proportion enables to achieve wideband absorption around $f_{\mathrm{H}}=3320 \mathrm{~Hz}$ while keeping an absorption coefficient greater than 0.5 below $1 \mathrm{kHz}$. Its input admittance is calculated as

$$
Y_{1}=\frac{S_{b} / S}{Z_{v}^{b} / \sigma+\mathrm{j} Z_{0} \cot \left(\tilde{k}_{0, R} D\right)}+\frac{S_{u} / S}{Z_{v}^{u} / \sigma+Z_{0}},
$$

with $Z_{v}^{u}$ (resp. $Z_{v}^{b}$ ) the transfer impedances of the unbacked (resp. backed) MCPs and $S_{u}$ (resp. $S_{b}$ ) their surface area such that $S_{u}+S_{b}=S$. The parameters constitutive of the unbacked and backed MCPs can be separately optimized to achieve maximum dissipation given their respective load conditions. This strategy has been used to provide the results of Fig. 10(b).

One now assumes that GCA I is backed by an open cavity of depth $D=145 \mathrm{~mm}$ and of the same radius than the impedance tube. Its input impedance then reads 
(a)

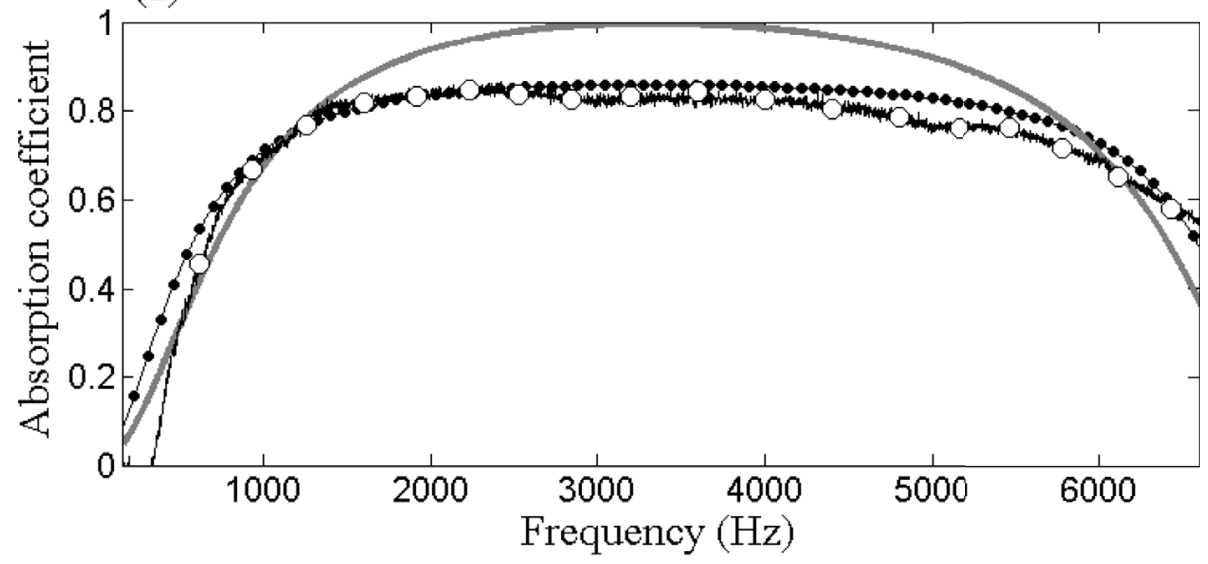

(b)

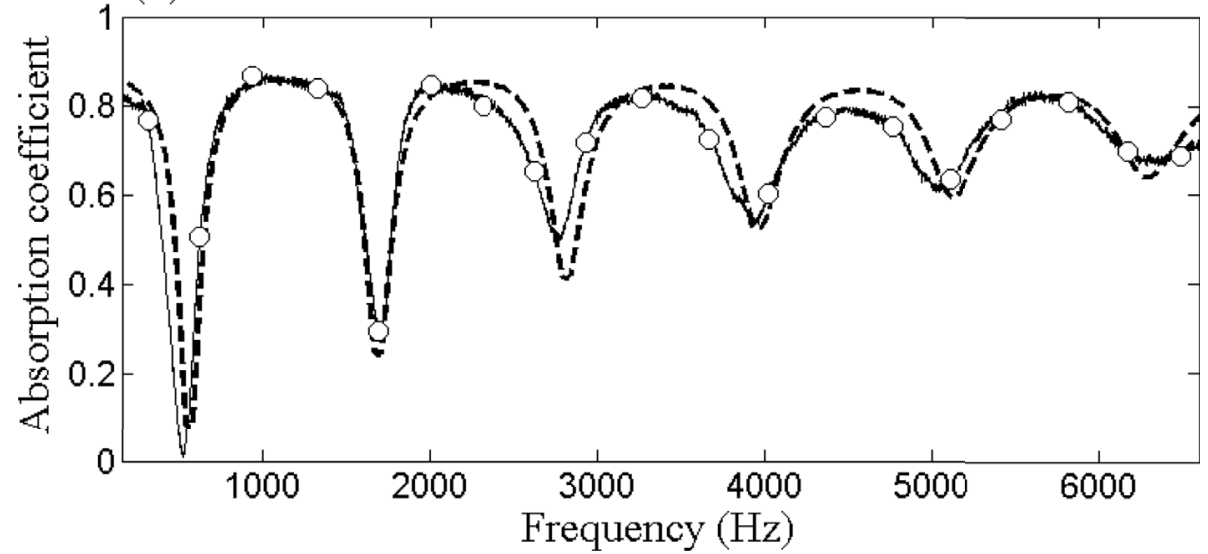

Fig. 9. Normal incidence absorption coefficient of GCA I when backed by cavities with rigid (a) and open (b) terminations, respectively located 24 mm and

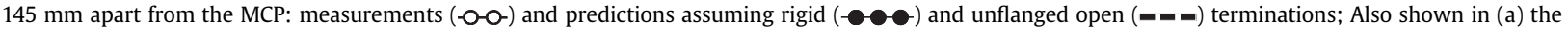
normal incidence absorption coefficient predicted for critically-coupled GCA I ( $)$ with back cavity and with channels radius increased to $17.5 \mu$ m.

$$
Z_{1}=\frac{Z_{v}}{\sigma}+Z_{0} \frac{r_{c}^{2}}{R^{2}} \frac{\left(1+r_{\text {open }}^{\prime}\right) \cos \left(\tilde{k}_{0, R} D\right)+\mathrm{j}\left(1-r_{\text {open }}^{\prime}\right) \sin \left(\tilde{k}_{0, R} D\right)}{\left(1+r_{\text {open }}^{\prime}\right) \sin \left(\tilde{k}_{0, R} D\right)+\mathrm{j}\left(1-r_{\text {open }}^{\prime}\right) \cos \left(\tilde{k}_{0, R} D\right)}
$$

The theoretical and experimental results shown in Fig. 9(b) confirm that the system ensures a constant maximum absorption value of about 0.85 except around the cavity quarter-wavelength resonance frequencies. At these frequencies, the normal air particle velocity is zero-valued over the MCP surface, leading to a significant drop in the absorption coefficient. However, the increase of radiation leakages with frequency at the unflanged cavity termination [see Fig. 7(a)] causes an increase of the absorption value at the dips when the resonance order increases. Although not shown, it was observed that, by decreasing the depth of the open cavity, one upshifts the first quarter-wavelength resonance frequency and the absorption spectrum tends towards that of an unbacked MCP with unflanged radiation condition that provides, as seen in Fig. 7(a), a "plateau" of high absorption over a broad bandwidth. It results from Figs. 7, 9 and 10 that the absorption performance of dissipative MCPs is highly sensitive to their load impedance. The sensitivity of the MCPs optimal properties to the load impedance is examined in Sec. 4.

\section{Optimisation study}

\subsection{Optimisation of MCPs under a general load}

From Sec. 2.3, it can be shown that the MCP specific input impedance, $z_{1, \beta}=Z_{1, \beta} / Z_{0}$, under a general specific load $\beta=\beta_{R}+$ $\mathrm{j} \beta_{I}, \beta_{R} \geq 0$, is well approximated by $r_{\mathrm{tr}}+\beta$ over a broad frequency range $(\mathrm{Sh}<1)$ with $r_{\mathrm{tr}}=R_{\mathrm{tr}} / Z_{0}, R_{\mathrm{tr}}$ being given by Eq. (24). 

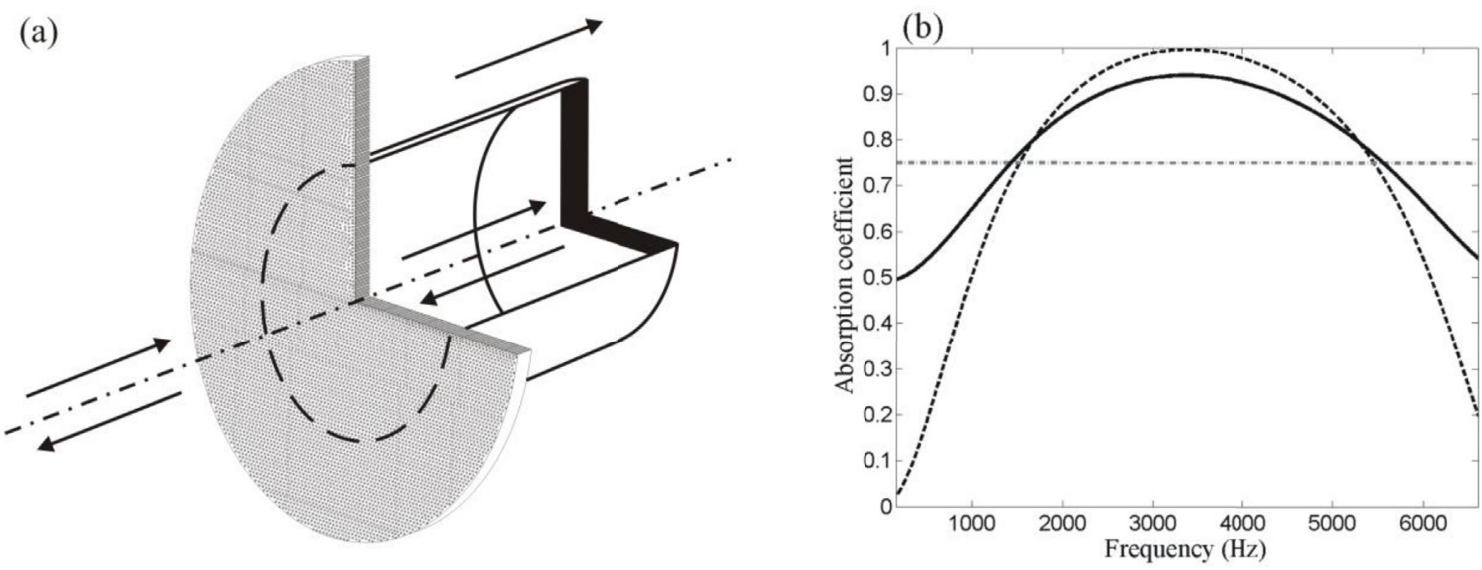

Fig. 10. (a) Quarter cut drawing of a hybrid absorber whose central part is a rigidly-backed MCP and annular part is an unbacked MCP; (b) Comparison between the predicted normal incidence absorption coefficients related to an optimal unbacked MCP ( $-\backsim)$ assuming plane wave anechoic load, a rigidly-backed critically-coupled MCP (- - with cavity depth $24 \mathrm{~mm}$ and a hybridized MCP ( $)$ made up of a parallel arrangement of the previous absorbers with equal surface proportion.

An optimisation study is now performed to find the optimal value of $r_{\mathrm{tr}}, r_{\mathrm{tr}, \beta}^{\mathrm{opt}}$, that maximizes the power dissipated by the MCP under normal plane wave incidence. The dissipated power defined in Sec. 2.3 can be recast as the following second-order equation in $r_{\mathrm{tr}}$

$$
\eta r_{\mathrm{tr}}^{2}-2\left[2-\eta\left(1+\beta_{R}\right)\right] r_{\mathrm{tr}}+\left[\eta|\beta|^{2}+2 \beta_{R}(\eta-2)+\eta+4\right]=0
$$

In order to have real positive solutions, its discriminant $\Delta=-4\left(\eta^{2} \beta_{I}^{2}+8 \eta-4\right)$ must be positive. $\Delta$ is a second-order polynomial in $\eta$ whose discriminant $\Delta^{\prime}=16\left(4+\beta_{I}^{2}\right)$ is always positive. $\Delta$ can thus be written as $\Delta=-4 \beta_{I}^{2}\left(\eta-\eta^{+}\right)\left(\eta-\eta^{-}\right)$with $\eta^{ \pm}=\left(4 / \beta_{I}^{2}\right)\left(-1 \pm \sqrt{1+\beta_{I}^{2} / 4}\right)$ for $\beta_{I} \neq 0$, the case $\beta_{I}=0$ being treated in Sec. 4.2 . Since $\eta^{-}<$ $0, \Delta \geq 0$ if and only if $0 \leq \eta \leq \eta^{+}$, so that the maximum dissipated power $\eta_{\beta}^{\text {opt }}=\eta^{+}$takes the form

$$
\eta_{\beta}^{\mathrm{opt}}=\frac{4}{\beta_{I}^{2}}\left(-1+\sqrt{1+\frac{\beta_{I}^{2}}{4}}\right)
$$

which does not depend on the load resistance, but only on its reactance.

When $\eta_{\beta}^{\text {opt }}=\eta^{+}, \Delta=0$ and the second-order equation in $r_{\text {tr }}$ admits a unique positive solution that provides the load dependence of the optimal MCP transfer resistance,

$$
r_{\mathrm{tr}, \beta}^{\mathrm{opt}}=\frac{2}{\eta_{\beta}^{\mathrm{opt}}}-\left(1+\beta_{R}\right)
$$

valid as long as $\left(1+\beta_{R}\right)<2 / \eta_{\beta}^{\text {opt }}$ for $r_{\mathrm{tr}, \beta}^{\text {opt }}$ to stay positive. Fig. 11(a) illustrates the load dependence of the optimal MCP transfer resistance. It can be used as a design chart to deduce the optimal MCP constitutive parameters from a given load.

Substituting Eqs. (29) and (30) into the expression, $z_{1, \beta}^{\mathrm{opt}}=r_{\mathrm{tr}, \beta}^{\mathrm{opt}}+\beta$, for the optimal MCP input impedance, one gets

$$
z_{1, \beta}^{\mathrm{opt}}=\frac{\beta_{I}^{2}}{-2+\sqrt{4+\beta_{I}^{2}}}-1+\mathrm{j} \beta_{I}
$$

which shows that $z_{1, \beta}^{\text {opt }}$ only depends on the load reactance, as it is the case for $\alpha_{\beta}^{\text {opt }}, \tau_{\beta}^{\prime \text { opt }}$ and $\eta_{\beta}^{\text {opt }}$. One appreciates from Fig. 11(b and c) the dependence of these quantities with respect to $\beta_{I}$. It indicates that the best dissipation performance that can be achieved by a MCP absorber is that under anechoic load conditions. 
(a)
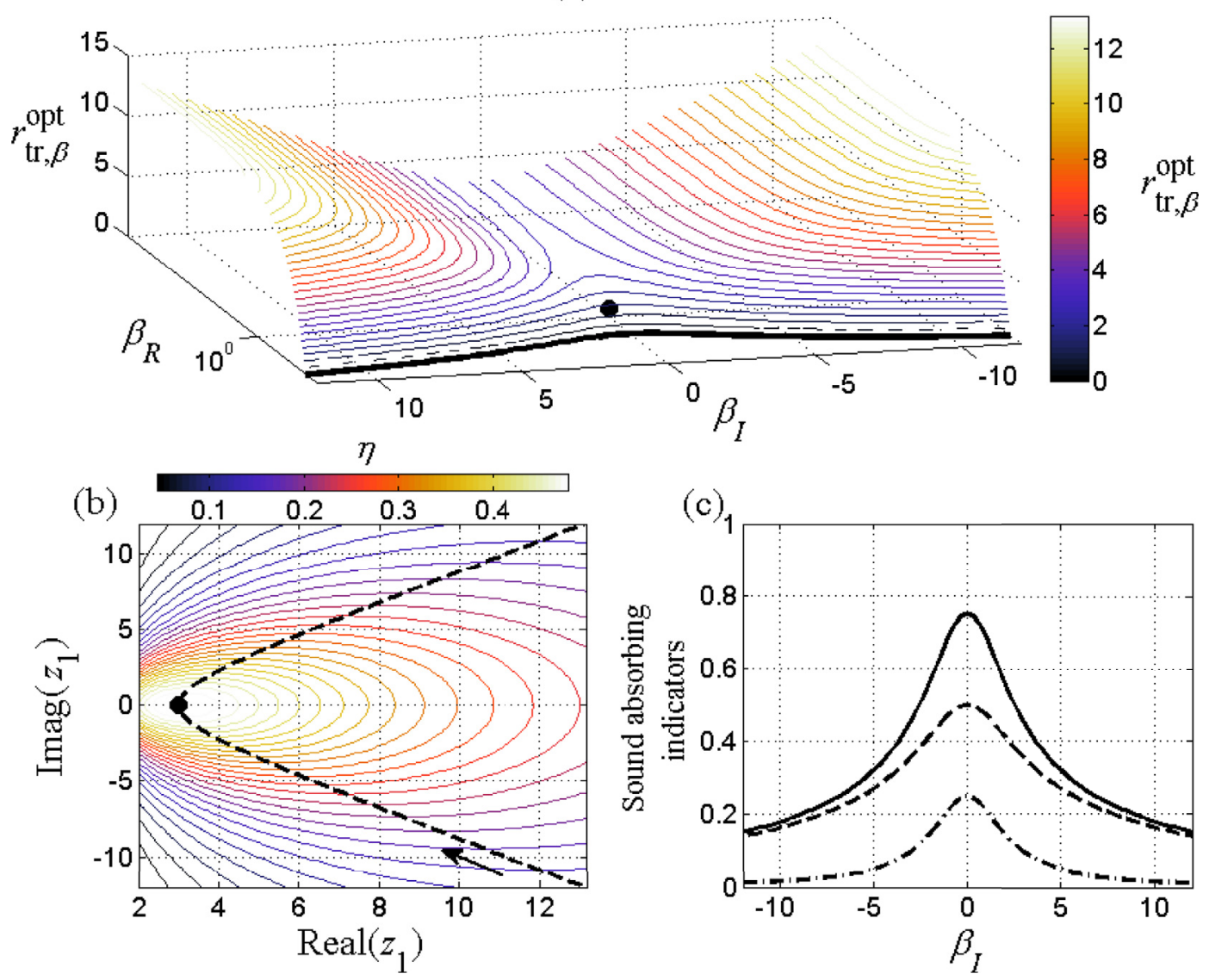

Fig. 11. (a) Adaptation of the MCP optimal transfer resistance, $r_{\operatorname{tr} \beta}^{\text {opt }}$, to the load specific impedance, $\beta$, to achieve maximal dissipation along with the maximum load resistance curve (-) above which $r_{\operatorname{tr}, \beta}^{\text {opt }}$ reaches negative unphysical values; (b) Phasor curve dependence of the optimal specific input impedance, $z_{1, \beta}^{\text {opt }}{ }_{1}$, to the load reactance, $-12 \leq \beta_{I} \leq 12$, along with circles of constant dissipation; (c) Dependence of the optimal absorption ( dissipation ( $-\mathbf{E}$ ) coefficients to the load reactance $\beta_{\mathrm{I}}$; also shown in (a) and (b) the optimal configuration assuming plane wave anechoic conditions ( ).

\subsection{Optimisation of MCPs assuming a plane wave anechoic load}

In practice, knowledge of the MCP load impedance may not be readily available. It is then of interest to examine the sensitivity of the MCP acoustical performance to the actual load, once it has been optimized assuming a plane wave anechoic load. The latter case corresponds to $\beta_{R}=1$ and $\beta_{I}=0$. The discriminant of Eq. (28) then simplifies into $\Delta=16(1-2 \eta)$. Real physical solutions require $\eta \leq 1 / 2$. In other words, the dissipation of a MCP with anechoic load cannot exceed 0.5 , as already observed in Fig. 2. In the optimal case $\eta=\eta^{\text {opt }}=1 / 2(\Delta=0)$, the unique solution to Eq. (28) is the optimal specific transfer resistance $r_{\mathrm{tr}, 1}^{\mathrm{opt}}=2$. Substituted in Eq. (24), the optimal channel radius reads

$$
a_{1}^{\mathrm{opt}}=2 \sqrt{\frac{t \mu}{\sigma Z_{0}\left(1-4 B_{v}\right)}}
$$

For the GCA I parameters reported in Table 1 , this leads to $a_{1}^{\mathrm{opt}}=13.2 \mu \mathrm{m}$ which is quite close to the actual value $12.5 \mu \mathrm{m}$. Hence, GCA I is nearly optimal, as anticipated from Figs. 2 and 3(a) and 7(a). For GCA II (resp. GCA III), this leads to $a_{1}^{\text {opt }}=$ $23.8 \mu \mathrm{m}$ (resp. $16.9 \mu \mathrm{m}$ ). These values are much higher than the actual ones, $5 \mu \mathrm{m}$ (resp. $12.5 \mu \mathrm{m}$ ). They provide too high resistance resulting in lower absorption values, as seen in Fig. 12, but also in Figs. 3(a) and Fig. 7(b).

One deduces from $r_{\mathrm{tr} 1}^{\mathrm{opt}}=2$ the optimal input impedance, absorption and transmission of MCPs under an anechoic load, namely $z_{1,1}^{\mathrm{opt}}=3, \alpha_{1}^{\mathrm{opt}}=3 / 4$ and $\tau_{1}^{\prime}{ }_{1}^{\mathrm{opt}}=1 / 4$. Higher values of the absorption coefficient could be obtained for less resistive MCPs, described by the smallest of the two solutions to Eq. (28), $r_{\mathrm{tr}, 1}^{-}$, with $r_{\mathrm{tr}, 1}^{ \pm}=2(1-\eta \pm \sqrt{1-2 \eta}) \eta^{-1}$ assuming $\Delta>0$ ( $\eta<$ $1 / 2$ ). However, as it can be seen from the grey curves in Fig. 12, this would be at the expense of lower dissipation and higher 
(a)

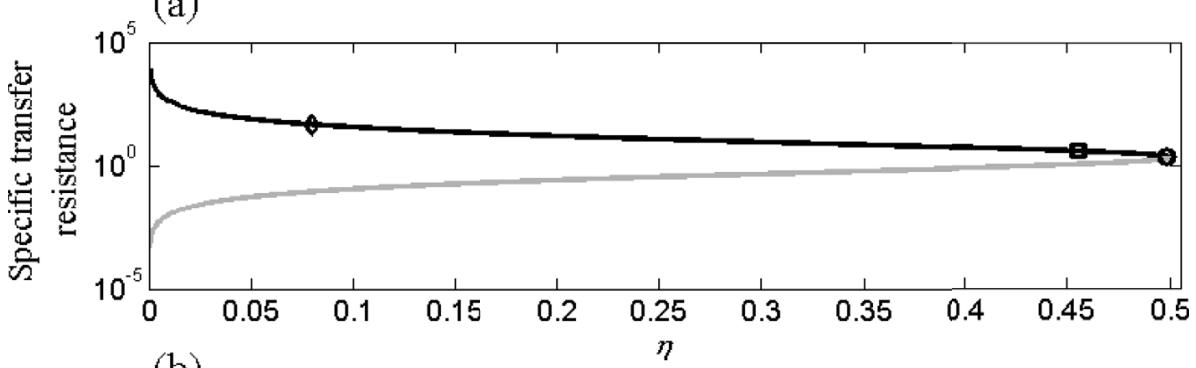

(b)

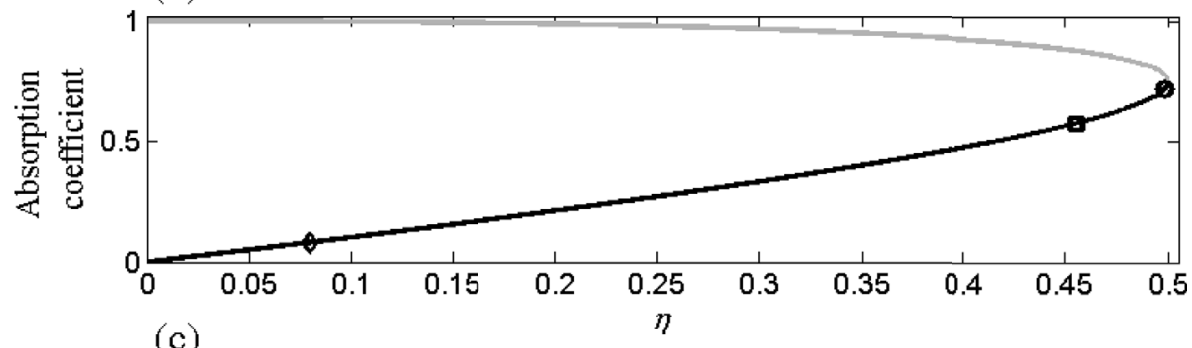

(c)

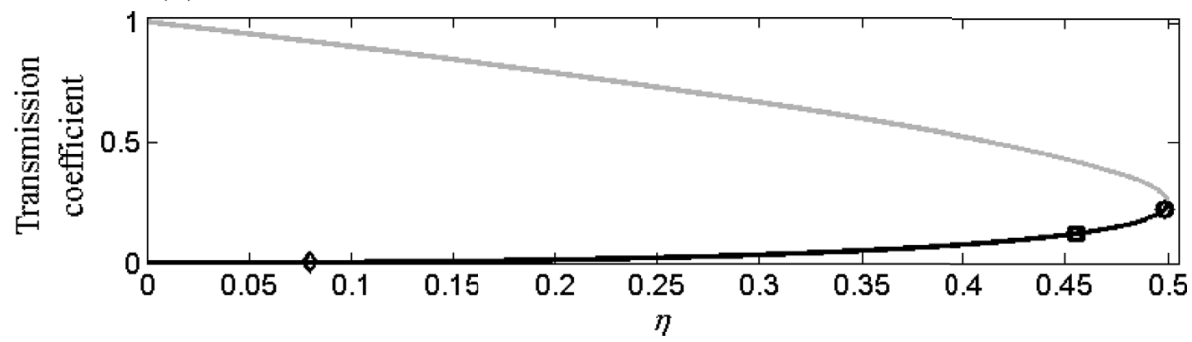

Fig. 12. Dependence of (a) the specific transfer resistances $\left(r_{\mathrm{tr}}^{1+}\right.$, black; $r_{\mathrm{tr}}^{1-}$, grey), (b) the absorption coefficient and (c) the transmission coefficient to the power $\eta$ dissipated by an unbacked MCP under an anechoic load; also shown are the acoustical properties of GCA I (circle), GCAII (diamond) and GCA III (square). An overview of the sensitivity of the absorption, transmission and dissipation coefficients of an optimized MCP to the load impedance is shown in Fig. 13.

transmission values. It corresponds to a class of acoustically transparent MCPs that could provide suitable candidates to design low-frequency anechoic terminations. The black curves in Fig. 12 correspond to another class of MCPs, of lesser interest, with high resistance, low transmission and which reflect a large part of the incident wave.

The MCP transfer resistance is optimized assuming an anechoic load, but its actual load is varied such that its specific input impedance, $z_{1, \beta}=r_{\mathrm{tr}, 1}^{\mathrm{opt}}+\beta$, encompasses all the types of loads encountered in Sec. 3, from purely reactive due to rigid backing $\left(\beta_{R}=0\right)$ to purely resistive due to anechoic conditions $\left(\beta_{I}=0\right)$. One retrieves in Fig. 13 the trends observed in the absorption spectra of Fig. 7(a) and Fig. (9) for which GCA I is nearly optimal under anechoic load.

\subsection{Extension to plane wave oblique incidence}

The MCP optimal channel radius is then dependent on the incidence angle $\theta$. It is readily obtained by substituting the MCP resistance, $r_{\mathrm{tr}}$, by its effective value $r_{\mathrm{tr}} \cos (\theta)$. Assuming an anechoic load and maximizing the MCP dissipation leads to

$$
a_{1}^{\theta \text { opt }}=2 \sqrt{\frac{t \mu \cos (\theta)}{\sigma Z_{0}\left(1-4 B_{\nu}\right)}}
$$

From Eq. (33), one observes that GCA I, II and III are optimal if they are respectively insonified under $24.7^{\circ}, 87.5^{\circ}$ and $57.0^{\circ}$ incidence angles. If the MCP is rigidly-backed by a cavity of depth $D$, maximizing the absorption coefficient, $\alpha_{D}^{\theta}=$ $4 r_{\text {tr }} \cos (\theta)\left\{\left[1+r_{\text {tr }} \cos (\theta)\right]^{2}+\cot ^{2}\left(k_{0} D \cos (\theta)\right)\right\}^{-1}$, leads to

$$
a_{D}^{\theta}{ }^{\mathrm{opt}}=a_{1}^{\theta} \mathrm{opt} \sqrt{2\left|\sin \left(k_{0} D \cos (\theta)\right)\right|}
$$

with $\sin \left(k_{0} D \cos (\theta)\right)$ replaced by $\sin \left(k_{0} D\right)$ in case of a locally-reacting honeycomb-filled cavity. 
(a)

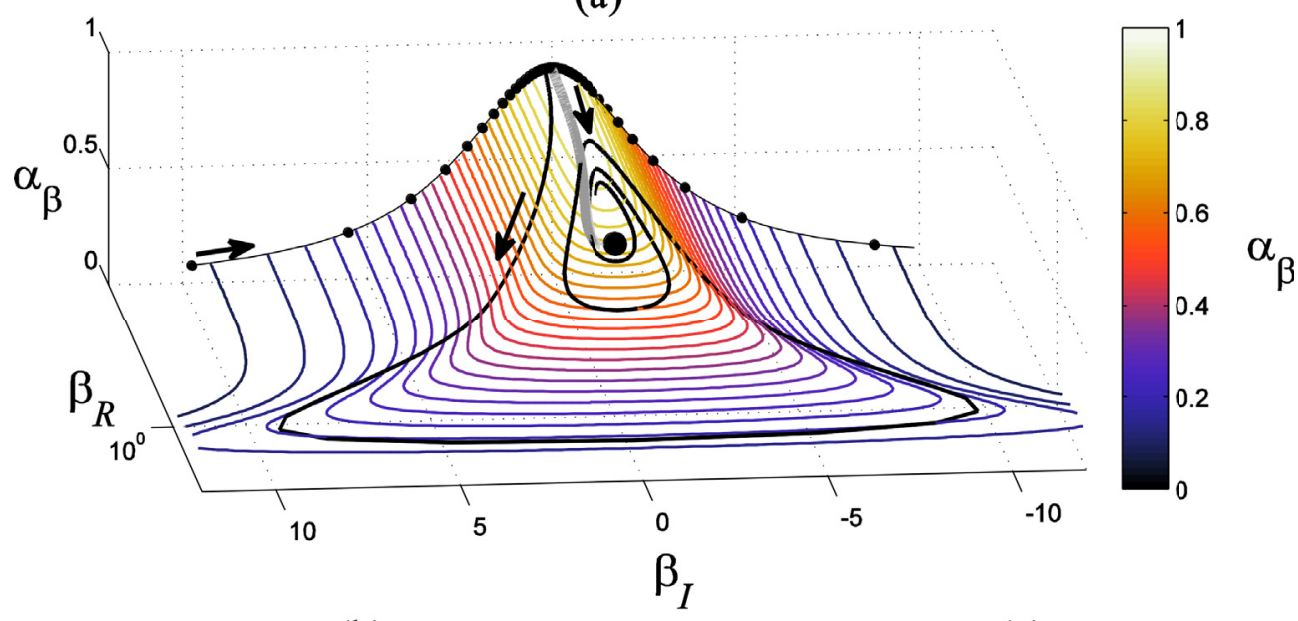

(b)

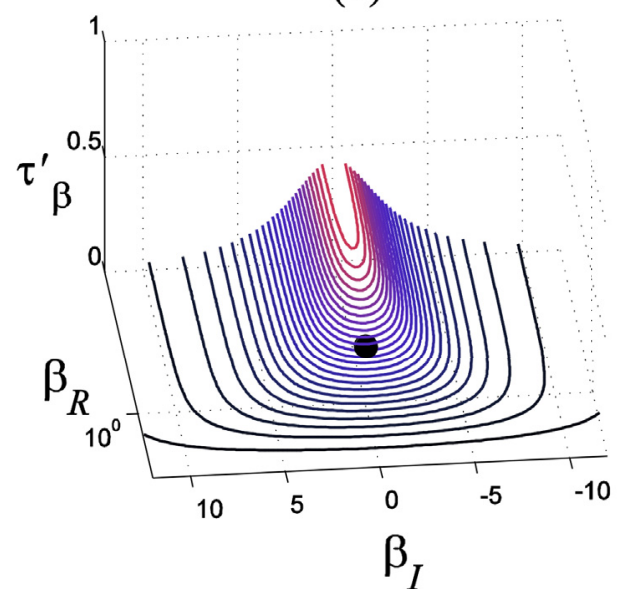

(c)

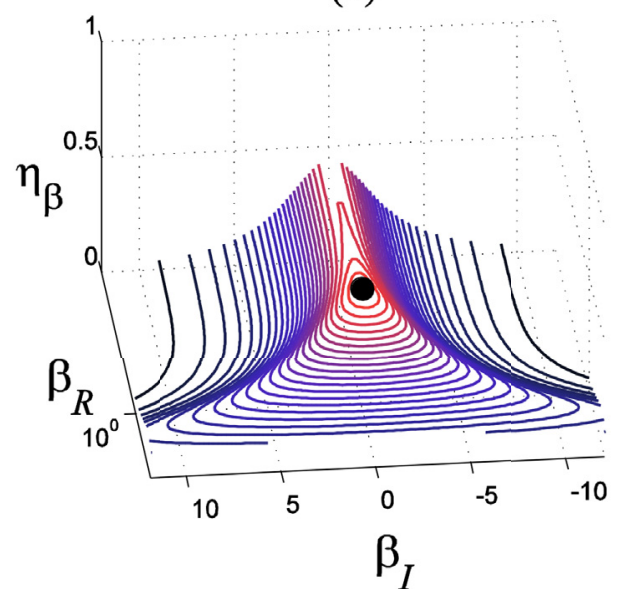

Fig. 13. Influence of the load specific impedance, $\beta$, on the absorption (a), transmission (b) and dissipation (c) coefficients of a MCP whose specific transfer resistance, $r_{\mathrm{tr} .1}^{\mathrm{opt}}=2$, is optimal under plane wave anechoic condition ( $)$; Also shown in (a) the locii of the absorption when the frequency varies from $160 \mathrm{~Hz}$ to $6600 \mathrm{~Hz}$ under a number of load impedances: rigid cavity of depth $24 \mathrm{~mm}$ (- - -), open cavity of depth $72 \mathrm{~mm}$ with unflanged termination ( unbacked MCP with flanged ( ${ }^{\prime}$ ) and unflanged $(\longleftarrow)$ radiation conditions.

These optimal values of the channel radii monotonically decrease when increasing $\theta$, except for a non-locally reacting cavity where this is observed only when $0<k_{0} D<\pi / 2(\bmod \pi)$. This confirms the trends seen in Fig. 5(b) and (c) for which optimal MCP performance occur for values of $\mathrm{Kn}$ in slip-flow regime that increase with the incidence angle.

\section{Conclusions}

Theoretical and experimental studies have been carried out to analyse sound propagation through MCP narrow channels in slip-flow regime, appropriate for large Knudsen numbers lower than 0.1. The ability of MCPs to dissipate the power due to normal and oblique incident plane waves has been optimized and the sensitivity of their optimal performance has been examined for a wide range of loading conditions, including unbacked and rigidly-backed configurations. The following conclusions have been drawn:

(1) Because MCPs have a Knudsen number Kn usually comprised between $10^{-3}$ and $10^{-1}$, their viscous transfer impedance and thermal admittance have been derived in the frame of the slip-flow regime. Theoretical modelling and FEM simulations showed that, in the broad frequency range over which MCPs exhibit narrow channel behaviour (Sh $<1)$, the dissipation is dominated by viscous effects and there is no thermal dissipation in the capillary channels. It was found that the slip-flow model significantly deviates from the continuum approach when evaluating the transfer impedance of MCPs with extremely small channels radius, e.g. for $\mathrm{Kn}>0.03$. But, for lower Kn and over a wide range of 
incidence angles $\left(\theta<80^{\circ}\right)$, the MCPs dissipation properties are well approximated by the continuum approach, up to a factor $\mathrm{Kn} \mathrm{Sh}^{2}$.

(2) Owing to their micrometric channels radius and high porosity, MCPs are pure resistive absorbers with constant resistance and minute reactance over a wide frequency band, the expression of which has been derived in terms of their constitutive parameters for $0.001<\mathrm{Kn}<0.1$. Theoretical and experimental studies showed the ability of unbacked optimal MCPs to achieve absorption values greater than 0.7 up to $k_{0} R=1.84$ with a flat absorption plateau of 0.86 up to $k_{0} R=0.3$ under normal incidence. MCPs could thus be used as suitable low frequency anechoic terminations for transmission tubes in the no-flow case and more generally as calibrated acoustic materials.

(3) Models and measurements showed that rigidly-backed MCPs are efficient wideband absorbers in the mid-high frequency range under normal incidence, with a half-bandwidth spanning 12 octaves around $k_{0} R=0.9$ for a 24 mm cavity depth. These absorbers can be critically-coupled by fine tuning of the MCP channels radius to achieve unity absorption at the Helmholtz reduced frequency. But they still suffer from poor low-frequency performance. A hybridized device made up of a parallel arrangement of backed and unbacked MCPs with equal surface proportion has been proposed that achieves, in theory, wideband absorption that stays above 0.5 at low frequencies.

(4) Optimal MCP channels radius were derived under general incidence angle assuming anechoic or rigid backing. Under normal incidence, MCPs optimal specific resistance $r_{\operatorname{tr} \beta}^{\text {opt }}$ was found to be highly sensitive to the load impedance $\beta$ and an exact relationship has been derived between these two quantities that can be used as a design chart. For instance, it was shown that $r_{\mathrm{tr}, \beta}^{\text {opt }}=2$ under an ideal anechoic load $(\beta=1)$ leading to a maximum dissipated power of 0.5 . But $r_{\mathrm{tr}, \beta}^{\text {opt }}$ can reach higher values under more resistive and/or reactive loads, leading to an optimal input impedance and a dissipation that only depend on the load reactance.

In summary, MCPs are suitable candidates as low-frequency broadband absorbers under a wide range of incidence angles. Their constitutive parameters can be tailored to achieve a targeted absorption value. In particular, a weakly-dissipative acoustically-transparent MCP could be designed to work as a thin anechoic termination in Kundt tubes at low frequencies. Rigidly-baked MCPs could also be of interest as optimized locally-reacting wall treatments in flow ducts due to their calibrated acoustic resistance that should then match that of a Cremer-type impedance at a given frequency in order to ensure maximum damping of the least attenuated duct mode [50]. Comparison of their performance against MPP liners would be instructive, but it would deserve a proper study in the frame of guided-wave propagation theory in flow duct with the axial attenuation per unit length as the relevant acoustical indicators. Finally, further study on the micro-capillaries hydrophobic properties could assess their potential use as sound absorbers in underwater applications.

\section{Declaration of competing interest}

The authors declare that they have no known competing financial interests or personal relationships that could have appeared to influence the work reported in this paper.

\section{CRediT authorship contribution statement}

Cédric Maury: Conceptualization, Investigation, Methodology, Data curation, Formal analysis, Writing - original draft, Writing - review \& editing. Teresa Bravo: Conceptualization, Investigation, Funding acquisition, Project administration, Validation, Methodology, Data curation, Formal analysis, Writing - original draft, Writing - review \& editing.

\section{Acknowledgments}

This study was funded in Spain by the Ministerio de Economía y Competitividad project TRA2017-87978-R, AEI/FEDER, UE, and the mobility program ILINK+2018. It was supported in France by the ANR VIRTECH (ANR-17-CE10-0012-01). The authors would like to thank J. Kergomard, Emeritus Director of Research at CNRS-LMA, for fruitful discussions, Dr. P. Ecker from GIDS $\mathrm{GmbH}$ for kindly borrowing us one of the MCPs and Dr. L. Sabatier, Research Engineer at CNRS-LMA, for X-ray imaging the MCPs.

\section{References}

[1] K. Sakagami, M. Morimoto, M. Yairi, Application of microperforated panel absorbers to room interior surfaces, Int. J. Acoust. Vib. 13 (2008) 120-124, https://doi.org/10.20855/ijav.2008.13.3229.

[2] F. Asdrubali, G. Pispola, Properties of transparent sound-absorbing panels for use in noise barriers, J. Acoust. Soc. Am. 121 (2007) 214-221, https://doi. org $/ 10.1121 / 1.2395916$.

[3] J. Kang, M.W. Brocklesby, Feasibility of applying micro-perforated absorbers in acoustic window systems, Appl. Acoust. 66 (2005) 669-689, https:// doi.org/10.1016/j.apacoust.2004.06.011.

[4] S. Allam, M. Åbom, A new type of muffler based on microperforated tubes, ASME J. Vib. Acoust. 133 (2011) 8, https://doi.org/10.1115/1.4002956, 031005.

[5] M.A. Temiz, J. Tournadre, I.L. Arteaga, A. Hirschberg, Non-linear acoustic transfer impedance of micro-perforated plates with circular orifices, J. Sound Vib. 366 (2016) 418-428, https://doi.org/10.1016/j.jsv.2015.12.022.

[6] D.Y. Maa, Potential of microperforated panel absorbers, J. Acoust. Soc. Am. 104 (1998) 2861-2866, https://doi.org/10.1121/1.423870. 
[7] D.Y. Maa, Microperforated panel wide band Absorbers, Noise Control Eng. J. 29 (1987) 77-84, https://doi.org/10.3397/1.2827694.

[8] J. Kang, H.V. Fuchs, Predicting the absorption of open weave textiles and micro- perforated membranes backed by an air space, J. Sound Vib. 220 (1999) 905-920, https://doi.org/10.1006/jsvi.1998.1977.

[9] K. Sakagami, K. Matsutani, M. Morimoto, Sound absorption of a double-leaf micro- perforated panel with an air-back cavity and a rigid back wall: detailed analysis with a Helmholtz-Kirchhoff integral formulation, Appl. Acoust. 71 (2010) 411-417, https://doi.org/10.1016/j.apacoust.2009.11.014.

[10] T. Bravo, C. Maury, Optimised micro-perforated panels for broadband absorptivity, in: 26th International Congress on Sound and Vibration (ICSV26) Montreal, 2019. Peer-reviewed Invited paper 486, https://iiav.org/archives_icsv/2019_icsv26/content/papers/papers/full_paper_486_ 20190128164618463.pdf.

[11] K. Sakagami, Y. Nagayama, M. Morimoto, M. Yairi, Pilot study on wideband sound absorber obtained by combination of two different microperforated panel (MPP) absorbers, Acoust Sci. Technol. 30 (2009) 154-156, https://doi.org/10.1250/ast.30.154.

[12] C. Wang, L. Huang, On the acoustic properties of parallel arrangement of multiple micro- perforated panel absorbers with different cavity depths, J. Acoust. Soc. Am. 130 (2011) 208-218, https://doi.org/10.1121/1.3596459.

[13] Y.J. Qian, J. Zhang, N. Sun, D.Y. Kong, X.X. Zhang, Pilot study on wideband sound absorber obtained by adopting a serial-parallel coupling manner, Appl. Acoust. 124 (2017) 48-51, https://doi.org/10.1016/j.apacoust.2017.03.021.

[14] H.-S. Kim, P.-S. Ma, B.-K. Kim, S.-R. Kim, S.-H. Lee, Low-frequency sound absorption of elastic micro-perforated plates in a parallel arrangement, J. Sound Vib. 460 (2019) 114884, https://doi.org/10.1016/j.jsv.2019.114884.

[15] D. Li, D. Chang, B. Liu, Enhanced low- to mid-frequency sound absorption using parallel-arranged perforated plates with extended tubes and porous materials, Appl. Acoust. 127 (2017) 316-323, https://doi.org/10.1016/j.apacoust.2017.06.019.

[16] F. Simon, Long elastic open neck acoustic resonator for low frequency absorption, J. Sound Vib. 421 (2018) 1-16, https://doi.org/10.1016/j.jsv.2018.01. 044.

[17] C. Zhang, X. Hu, Three-dimensional single-port labyrinthine acoustic metamaterial: perfect absorption with large bandwidth and tunability, Phys. Rev. Appl. 6 (2016), 064025, https://doi.org/10.1103/PhysRevApplied.6.064025.

[18] M. Yang, S. Chen, C. Fu, P. Sheng, Optimal sound-absorbing structures, Mater. Horiz. 4 (2017) 673-680, https://doi.org/10.1039/C7MH00129K.

[19] B. Fenech, G.M. Keith, F. Jacobsen, The use of microperforated plates to attenuate cavity resonances, J. Acoust. Soc. Am. 120 (2006) 1851-1858, https:/I doi.org/10.1121/1.2258438.

[20] K. Sakagami, M. Morimoto, W. Koike, A numerical study of double-leaf microperforated panel absorbers, Appl. Acoust. 67 (2006) 609-619, https://doi. org/10.1016/j.apacoust.2005.11.001.

[21] S. Sack, M. Åbom, Modal filters for mitigation of in-duct sound, Proc. Mtgs. Acoust. 29 (2016), 040004, https://doi.org/10.1121/2.0000473.

[22] C. Maury, T. Bravo, D. Mazzoni, The use of micro-perforations to attenuate the cavity pressure fluctuations induced by a low-speed flow, J. Sound Vib. 439 (2019) 1-16, https://doi.org/10.1016/j.jsv.2018.09.045.

[23] J.P. Arenas, M.J. Crocker, Recent Trends in Porous Sound Absorbing Materials, Sound \& Vib., July 2010. Materials Reference Issue, 12-17, http://www. sandv.com/downloads/1007croc.pdf.

[24] Y. Zheng, W. Dai, D. Ryan, H. Wu, Fabrication of freestanding microperforated membranes and their applications in microfluidics, Biomicrofluidics 4 (2010), 036504, https://doi.org/10.1063/1.3491474.

[25] Y.J. Qian, D.Y. Kong, S.M. Liu, S.M. Sun, Z. Zhao, Investigation on micro-perforated panel absorber with ultra-microperforations, Appl. Acoust. 74 (2013) 931-935, https://doi.org/10.1016/j.apacoust.2013.01.009.

[26] T. Bravo, C. Maury, C. Pinhède, Enhancing sound absorption and transmission through flexible multi-layer micro-perforated structures, J. Acoust. Soc. Am. 134 (2013) 3663-3673, https://doi.org/10.1121/1.4821215.

[27] G. Pispola, K.V. Horoshenkov, F. Asdrubali, Transmission loss measurement of consolidated granular media (L), J. Acoust. Soc. Am. 117 (2005) 2716-2719, https://doi.org/10.1121/1.1886365.

[28] M. Åbom, Measurement of the scattering-matrix of acoustical two-ports, Mech. Syst. Signal Process. 5 (1991) 89-104, https://doi.org/10.1016/08883270(91)90017-Y.

[29] T.E. Vigran, K. Kelders, W. Lauriks, P. Leclaire, T.F. Johansen, Prediction and measurements of the influence of boundary conditions in a standing wave tube, Acta Acustica united Acustica 83 (1997) 419-423.

[30] J.-P. Dalmont, E. Portier, Optimisation of anechoic duct termination using line theory, Appl. Acoust. 117 (2017) 141-144, https://doi.org/10.1016/j. apacoust.2016.10.024.

[31] K. Verdière, R. Panneton, S. Elkoun, T. Dupont, P. Leclaire, Transfer matrix method applied to the parallel assembly of sound absorbing materials, J. Acoust. Soc. Am. 134 (2013) 4648-4658, https://doi.org/10.1121/1.4824839.

[32] J.-P. Dalmont, J. Kergomard, X. Meynial, Realization of an anechoic termination for sound ducts at low frequencies, in: C. R. Acad. Sci. Paris 309 II, 1989 , pp. 453-458 (text in French, includes abridged English version). Downloaded the 11/10/2019 from, https://gallica.bnf.fr/ark:/12148/bpt6k56640789/ f459.image.

[33] C. Zwicker, C.W. Kosten, Sound Absorbing Materials, Elsevier Publishing Company, Inc., New-York, 1949 (reprinted by the Acoustical Society of the Netherlands, NAG).

[34] N.G. Hadjiconstantinou, Sound wave propagation in transition-regime micro- and nanochannels, Phys. Fluids 14 (2002) 802-809, https://doi.org/10 $1063 / 1.1431243$.

[35] V.F. Kozlov, A.V. Fedorov, N.D. Malmuth, Acoustic properties of rarefied gases inside pores of simple geometries, J. Acoust. Soc. Am. 117 (2005) 3402-3412, https://doi.org/10.1121/1.1893428.

[36] S. Kandlikar, S. Garimella, D. Li, S. Colin, M.R. King, Heat Transfer and Fluid Flow in Minichanels and Microchannels, second ed., Elsevier Ltd., Oxford, 2014 https://doi.org/10.1016/C2011-0-07521-X.

[37] M. Gad-el-Hak, The fluid mechanics of microdevices - the freeman scholar lecture, J. Fluid Eng. 121 (1999) 5-33, https://doi.org/10.1115/1.2822013.

[38] J.C. Maxwell, On stresses in rarefied gases arising from inequalities of temperature, Philos. Trans. Roy. Soc. Part I 170 (1879) 231-256. https://www. jstor.org/stable/109283.

[39] von M. Smoluchowski, Ueber Wärmeleitung in verdünnten gasen, Ann. Phys. Chem. 64 (1898) 101-130, https://doi.org/10.1002/andp.18983000110.

[40] E.H. Kennard, Kinetic Theory of Gases, McGraw-Hill Publishing Company, Inc., London, 1938, https://doi.org/10.1002/jctb.5000573907.

[41] A. Agrawal, S.V. Prabhu, Survey on measurement of tangential momentum accommodation coefficient, J. Vac. Sci. Technol., A 26 (2008) 634-645, https://doi.org/10.1116/1.2943641.

[42] T.H. Melling, The acoustic impedance of perforates at medium and high sound pressure levels, J. Sound Vib. 29 (1973) 1-65, https://doi.org/10.1016 S0022-460X(73)80125-7.

[43] M.R. Stinson, The propagation of plane sound waves in narrow and wide circular tubes, and generalization to uniform tubes of arbitrary crosssectional shape, J. Acoust. Soc. Am. 89 (1991) 550-558, https://doi.org/10.1121/1.400379.

[44] G. Karniadakis, A. Beskok, N. Aluru, Microflows and Nanoflows, Interdisciplinary Applied Mathematics Series, vol. 29, Springer, New-York, 2005.

[45] ASTM E1050-12, Standard Test Method for Impedance and Absorption of Acoustical Materials Using a Tube, Two Microphones and a Digital Frequency Analysis System, ASTM International, West Conshohocken, U.S.A, 2012. https://www.astm.org/Standards/E1050.htm.

[46] F. Silva, Ph. Guillemain, J. Kergomard, B. Mallaroni, A. Norris, Approximation formulae for the acoustic radiation impedance of a cylindrical pipe, J. Sound Vib. 322 (2009) 255-263, https://doi.org/10.1016/j.jsv.2008.11.008.

[47] H.E. de Bree, An overview of the microflown technologies, Acta Acustica Acustica 89 (2003) 163-172. Downloaded the 11/10/2019 from, https://www. ingentaconnect.com/content/dav/aaua/2003/00000089/00000001/art00018. 
[48] W.E. Zorumski, Generalized radiation impedance and reflection coefficients of circular and annular ducts, J. Acoust. Soc. Am. 54 (1973) 1667-1673, https://doi.org/10.1121/1.1914466.

[49] V. Romero-Garcia, G. Theocharis, O. Richoux, V. Pagneux, Use of complex frequency plane to design broadband and sub-wavelength absorbers, J. Acoust. Soc. Am. 139 (2016) 3395-3403, https://doi.org/10.1121/1.4950708.

[50] R. Kabral, L. Du, M. Åbom, Optimum sound attenuation in flow ducts based on the "exact" Cremer impedance, Acta Acustica united Acustica 102 (2016) 851-860, https://doi.org/10.3813/AAA.918999. 